Research Article; Received:December 28, 2020; Accepted: June 7, 2021

\title{
NEW INTEGRAL TYPE INEQUALITIES VIA RAINA-CONVEX FUNCTIONS AND ITS APPLICATIONS
}

\author{
Saad Ihsan BUTT ${ }^{1}$, Muhammad NADEEM ${ }^{1}$, Muhammad TARIQ $^{1}$ and Adnan ASLAM ${ }^{2}$ \\ 1 COMSATS University Islamabad, Lahore Campus, PAKISTAN \\ ${ }^{2}$ Department of Natural Science and Humanities, University of Engineering and Technology, \\ Lahore(RCET), PAKISTAN
}

\begin{abstract}
In this work, we discuss and introduce the novel literature about Raina-convex function and its algebraic properties. In addition, we elaborate and investigate Hermite-Hadamard and Fejér-type inequalities for newly discussed definition. Finally, using the newly introduced definition, we find and prove amazing new integral type inequalities and applications for mean to positive real numbers. The amazing techniques and wonderful ideas of this paper may inspire and motivate for further activities and research in this direction furthermore.
\end{abstract}

\section{INTRODUCTION}

During the whole of the $20^{\text {th }}$ century, an enormous and extreme research activity was done and fruitful ideas and magnificent results were obtained in mathematical analysis, functional analysis , convex analysis, mathematical economics and nonlinear optimization. But interesting and tremendous book namely "Inequalities", which is written by Hardy, Littlewood and Polya. This book has played an elegant role in popularization and importance of the subject of convex functions. The modern and amazing viewpoint on convexity entails a powerful, enlighten and distinguish interaction between analysis and geometry, which makes and enables the readers to shear a sense of excitement. The theory of convexity encompasses a large variety of classes of convex functions like functions, $s$-convex, $p$-convex, log-convex, $h$-convex, quasi convex and exponential type convex functions while it

2020 Mathematics Subject Classification. Primary: 26A51; Secondary: 26A33, 26D07, 26D10, $26 \mathrm{D} 15$.

Keywords. Hermite-Hadamard inequality, Hölder's inequality, power mean inequality, Rainaconvex function.

@ saadihsanbutt@gmail.com-Corresponding author; muhammadnadeem98847@gmail.com; captaintariq2187@gmail.com; adnanaslam15@yahoo.com

(D) 0000-0001-7192-8269; 0000-0003-2714-4314; 0000-0002-2078-0652; 0000-0003-4523-8023.

Communications Faculty of Sciences University of Ankara Series A1 (C)2021 Ankara University 
is good to understand and what they have in common, it is of equal importance to look inside their own field. The theory of convexity also played a magnificent act in the advances of theory of inequalities. Inequalities have a lot of applications in statistical problems, probability and numerical quadrature formulas. Due to rich and paramount history, convex analysis and inequalities have become an attractive, interesting and absorbing field for the researchers and for the attention of the reader, see $1,3,4,8,9,16,18,22]$.

In recent years, many researchers working in the direction of convexity and generalized convexity of Raina type using meaningful ideas and magnificent techniques to bring a new dimension to mathematical analysis and applied mathematics with different features in the literature. Interested readers see the references [2,7, 14, 15]. So that is the main aim and motivation of our work. Before we start, we need the following necessary known definitions and literature and throughout the paper, "( $\mathrm{H}-\mathrm{H})$ " means Hermite-Hadamard inequality and "diff mapp" means differential mapping.

\section{Preliminaries}

In this section we recall some basic definitions.

Definition 1. [10] $A \zeta: \mathscr{H} \rightarrow \mathscr{R}$ is called convex, if

$$
\zeta\left(\kappa \tau_{1}+(1-\kappa) \tau_{2}\right) \leq \kappa \zeta\left(\tau_{1}\right)+(1-\kappa) \zeta\left(\tau_{2}\right),
$$

holds $\forall \tau_{1}, \tau_{2} \in \mathscr{H}$ and $\kappa \in[0,1]$.

The well known and remarkable inequality concerning convex function is HermiteHadamard inequality given as:

Theorem 1. [6] If $\zeta: \mathscr{H}=\left[\tau_{1}, \tau_{2}\right] \rightarrow \mathscr{R}$ is a convex function, then

$$
\zeta\left(\frac{\tau_{1}+\tau_{2}}{2}\right) \leq \frac{1}{\tau_{2}-\tau_{1}} \int_{\tau_{1}}^{\tau_{2}} \zeta(\nu) d \nu \leq \frac{\zeta\left(\tau_{1}\right)+\zeta\left(\tau_{2}\right)}{2}
$$

The double inequality 2 is in reverse order if $\zeta$ is a concave function.

Theorem 2. If $\zeta: \mathscr{H}=\left[\tau_{1}, \tau_{2}\right] \rightarrow \mathscr{R}$ is a convex function, then

$$
\zeta\left(\frac{\tau_{1}+\tau_{2}}{2}\right) \int_{\tau_{1}}^{\tau_{2}} \xi(\nu) d \nu \leq \int_{\tau_{1}}^{\tau_{2}} \zeta(\nu) \xi(\nu) d \nu \leq \frac{\zeta\left(\tau_{1}\right)+\zeta\left(\tau_{2}\right)}{2} \int_{\tau_{1}}^{\tau_{2}} \xi(\nu) d \nu .
$$

In 1906, L. Fejér 5 proved the above integral inequality (3) which is known in the literature as Fejér inequality. Since the researchers have shown interest in the above inequality and as a result, various generalizations and improvements have have been appeared in the literature. This inequality has remained an area of great and vital field for research activities due to its widespread views and robustness 
applications in the field of mathematical and convex analysis.

In 2005, Raina [12] introduced a class of functions defined formally by

$$
\mathcal{F}_{\varkappa, \lambda}^{\aleph}(z)=\mathcal{F}_{\varkappa, \lambda}^{\aleph(0), \aleph(1), \ldots}(z)=\sum_{k=0}^{+\infty} \frac{\aleph(k)}{\Gamma(\varkappa k+\lambda)} z^{k},
$$

where $\aleph=(\aleph(0), \ldots, \aleph(k), \ldots)$ and $\varkappa, \lambda>0,|z|<\mathscr{R}$.

If $\varkappa=1, \lambda=0$ and $\aleph(k)=\frac{(\alpha)_{k}(\beta)_{k}}{(\gamma)_{k}}$ for $k=0,1,2, \ldots$, where $\alpha, \beta$ and $\gamma$ are parameters which can take arbitrary real or complex values (provided that $\gamma \neq$ $0,-1,-2, \ldots)$, and the symbol $\alpha_{k}$ denote the quantity

$$
(\alpha)_{k}=\frac{\Gamma(\alpha+k)}{\Gamma(\alpha)}=\alpha(\alpha+1) \ldots(\alpha+k-1), \quad k=0,1,2, \ldots,
$$

and restrict its domain to $|z| \leq 1$ (with $z \in \mathbb{C}$ ), then we have the classical hypergeometric function, that is

$$
\mathcal{F}(\alpha, \beta ; \gamma ; z)=\sum_{k=0}^{+\infty} \frac{(\alpha)_{k}(\beta)_{k}}{k !(\gamma)_{k}} z^{k}
$$

Also, if $\aleph=(1,1, \ldots)$ with $\varkappa=\alpha,(\operatorname{Re}(\alpha)>0), \lambda=1$, then

$$
E_{\alpha}(z)=\sum_{k=0}^{+\infty} \frac{z^{k}}{\Gamma(1+\alpha k)}
$$

The above function is called a classical Mittag-Leffler function.

Theorem 3. 11] Suppose $\zeta: \mathscr{H} \subseteq[0, \infty) \rightarrow \mathscr{R}$ be a diff mapp on $\mathscr{H}^{\circ}$ of $\mathscr{H}$ such that $\zeta^{\prime \prime} \in L^{1}\left[\tau_{1}, \tau_{2}\right]$, where $\tau_{1}, \tau_{2} \in \mathscr{H}$ with $\tau_{1}<\tau_{2}$. If $|\zeta|$ is convex on $\left[\tau_{1}, \tau_{2}\right]$, then$$
\left|\zeta\left(\frac{\tau_{1}+\tau_{2}}{2}\right)-\frac{1}{\tau_{2}-\tau_{1}} \int_{\tau_{1}}^{\tau_{2}} \zeta(\nu) d \nu\right| \leq \frac{\left(\tau_{2}-\tau_{1}\right)^{2}}{192}\left\{\left|\zeta^{\prime \prime}\left(\tau_{1}\right)\right|+6\left|\zeta^{\prime \prime}\left(\frac{\tau_{1}+\tau_{2}}{2}\right)\right|+\left|\zeta^{\prime \prime}\left(\tau_{2}\right)\right|\right\} .
$$

Theorem 4. [11] Suppose $\zeta: \mathscr{H} \subseteq[0, \infty) \rightarrow \mathscr{R}$ be a diff mapp on $\mathscr{H}^{\circ}$ such that $\zeta^{\prime \prime} \in L^{1}\left[\tau_{1}, \tau_{2}\right]$, where $\tau_{1}, \tau_{2} \in \mathscr{H}$ with $\tau_{1}<\tau_{2}$. If $\left|\zeta^{\prime \prime}\right|^{\ell}$ for $\ell \geq 1$ is convex on $\left[\tau_{1}, \tau_{2}\right]$, then

$$
\begin{aligned}
& \left|\zeta\left(\frac{\tau_{1}+\tau_{2}}{2}\right)-\frac{1}{\tau_{2}-\tau_{1}} \int_{\tau_{1}}^{\tau_{2}} \zeta(\nu) d \nu\right| \leq \frac{\left(\tau_{2}-\tau_{1}\right)^{2}}{48}\left(\frac{3}{4}\right)^{\frac{1}{\ell}} \\
& \quad \times\left\{\left(\frac{\left|\zeta^{\prime \prime}\left(\tau_{1}\right)\right|^{\ell}}{3}+\left|\zeta^{\prime \prime}\left(\frac{\tau_{1}+\tau_{2}}{2}\right)\right|^{\ell}\right)^{\frac{1}{\ell}}+\left(\left|\zeta^{\prime \prime}\left(\frac{\tau_{1}+\tau_{2}}{2}\right)\right|^{\ell}+\frac{\left|\zeta^{\prime \prime}\left(\tau_{2}\right)\right|^{\ell}}{3}\right)^{\frac{1}{\ell}}\right\} .
\end{aligned}
$$


Lemma 1. [17] Suppose $\zeta: \mathscr{H} \subseteq \mathscr{R} \rightarrow \mathscr{R}$ be a diff mapp on $\mathscr{H}^{\circ}$ such that $\zeta^{\prime} \in L^{1}\left[\tau_{1}, \tau_{2}\right]$, where $\tau_{1}, \tau_{2} \in \mathscr{H}$ with $\tau_{1}<\tau_{2}$. If $\alpha, \beta \in \mathscr{R}$, then

$$
\frac{\alpha \zeta\left(\tau_{1}\right)+\beta \zeta\left(\tau_{2}\right)}{2}+\frac{2-\alpha-\beta}{2} \zeta\left(\frac{\tau_{1}+\tau_{2}}{2}\right)-\frac{1}{\tau_{2}-\tau_{1}} \int_{\tau_{1}}^{\tau_{2}} \zeta(\nu) d \nu=\frac{\tau_{2}-\tau_{1}}{4}
$$

$$
\times \int_{0}^{1}\left[(1-\alpha-\kappa) \zeta^{\prime}\left(\kappa \tau_{1}+(1-\kappa) \frac{\tau_{1}+\tau_{2}}{2}\right)+(\beta-\kappa) \zeta^{\prime}\left(\kappa \frac{\tau_{1}+\tau_{2}}{2}+(1-\kappa) \tau_{2}\right)\right] d \kappa .
$$

Lemma 2. 17 For $\mathscr{L}>0$ and $0 \leq \varepsilon \leq 1$, we have

$$
\begin{gathered}
\int_{0}^{1}|\varepsilon-\kappa|^{\mathscr{L}} d \kappa=\frac{\varepsilon^{\mathscr{L}+1}+(1-\varepsilon)^{\mathscr{L}+1}}{\mathscr{L}+1} \\
\int_{0}^{1} \kappa|\varepsilon-\kappa|^{\mathscr{L}} d \kappa=\frac{\varepsilon^{\mathscr{L}+2}+(\mathscr{L}+1+\varepsilon)(1-\varepsilon)^{\mathscr{L}+1}}{(\mathscr{L}+1)(\mathscr{L}+2)}
\end{gathered}
$$

Owing to the aforementioned trend and inspired by the ongoing activities in this absorbing field, we organize the paper in the following pattern. Firstly, we introduce Raina-convex function and its properties. Secondly, we debate and investigate $(\mathrm{H}-$ H) and Fejér-type integral inequalities for Riana-convex functions. Furthermore, we find integral inequalities and applications about fractional calculus regarding Riana-convex functions.

\section{RAina-Convex FUnCtions AND its PROperties}

In this section we are going to add a new definition namely Raina-convex function and will study some of their algebraic properties..

Definition 2. A function $\zeta: \mathscr{H} \rightarrow \mathscr{R}$ is said to be Raina-convex function on $\mathscr{H}$, if the following inequality

$$
\zeta\left(\kappa \tau_{1}+(1-\kappa) \tau_{2}\right) \leq \zeta\left(\tau_{2}\right)+\kappa \mathcal{F}_{\varkappa, \lambda}^{\aleph}\left(\zeta\left(\tau_{1}\right)-\zeta\left(\tau_{2}\right)\right)
$$

holds $\forall\left[\tau_{1}, \tau_{2}\right] \in \mathscr{H}$ and $\kappa \in[0,1]$, where $\varkappa, \lambda>0$ and $\aleph=(\aleph(1), \aleph(2), \ldots, \aleph(\kappa))$ is a bounded sequence of positive real no.

Note that when we choose $\mathcal{F}_{\varkappa, \lambda}^{\aleph}\left(\zeta\left(\tau_{1}\right)-\zeta\left(\tau_{2}\right)\right)=\zeta\left(\tau_{1}\right)-\zeta\left(\tau_{2}\right)$, then Rainaconvex function collapse to the classical convex function.

Theorem 5. Let $\zeta, \xi: \mathscr{H}=\left[\tau_{1}, \tau_{2}\right] \rightarrow \mathscr{R}$. If $\zeta$ and $\xi$ are Raina-convex functions then

(i) $\zeta+\xi$ is Raina-convex function.

(ii) For $c \in \mathscr{R}$ and $(c \geq 0)$ then $c \zeta$ is Raina-convex function.

Proof. (i) Let $\zeta$ and $\xi$ be a Raina-convex functions, then

$$
\begin{aligned}
& (\zeta+\xi)\left(\kappa \tau_{1}+(1-\kappa) \tau_{2}\right) \\
& =\zeta\left(\kappa \tau_{1}+(1-\kappa) \tau_{2}\right)+\xi\left(\kappa \tau_{1}+(1-\kappa) \tau_{2}\right) \\
& \leq \zeta\left(\tau_{2}\right)+\kappa \mathcal{F}_{\varkappa, \lambda}^{\aleph}\left(\zeta\left(\tau_{1}\right)-\zeta\left(\tau_{2}\right)\right)+\xi\left(\tau_{2}\right)+\kappa \mathcal{F}_{\varkappa, \lambda}^{\aleph}\left(\xi\left(\tau_{1}\right)-\xi\left(\tau_{2}\right)\right)
\end{aligned}
$$




$$
\leq(\zeta+\xi)\left(\tau_{2}\right)+\kappa \mathcal{F}_{\varkappa, \lambda}^{\aleph}\left((\zeta+\xi)\left(\tau_{1}\right)-(\zeta+\xi)\left(\tau_{2}\right)\right)
$$

(ii) Let $\zeta$ be a Raina-convex function and $c \in \mathscr{R}$, then

$$
\begin{aligned}
& (c \zeta)\left(\kappa \tau_{1}+(1-\kappa) \tau_{2}\right) \\
& =c\left[\zeta\left(\kappa \tau_{1}+(1-\kappa) \tau_{2}\right)\right] \\
& \leq c\left[\zeta\left(\tau_{2}\right)+\kappa \mathcal{F}_{\varkappa, \lambda}^{\aleph}\left(\zeta\left(\tau_{1}\right)-\zeta\left(\tau_{2}\right)\right)\right] \\
& \leq(c \zeta)\left(\tau_{2}\right)+\kappa \mathcal{F}_{\varkappa, \lambda}^{\aleph}\left((c \zeta)\left(\tau_{1}\right)-(c \zeta)\left(\tau_{2}\right)\right),
\end{aligned}
$$

which completes the proof.

Theorem 6. Let $\zeta: \mathscr{H} \rightarrow \mathscr{J}$ be a Raina-convex function and $\xi: \mathscr{J} \rightarrow \mathscr{R}$ is non-decreasing function. Then $\xi \circ \zeta: \mathscr{H} \rightarrow \mathscr{R}$ is Raina-convex function.

Proof. $\forall \tau_{1}, \tau_{2} \in \mathscr{H}$ and $\kappa \in[0,1]$, we have

$$
\begin{aligned}
& (\xi \circ \zeta)\left(\kappa \tau_{1}+(1-\kappa) \tau_{2}\right) \\
& =\xi\left(\zeta\left(\kappa \tau_{1}+(1-\kappa) \tau_{2}\right)\right) \\
& \leq \xi\left[\zeta\left(\tau_{2}\right)+\kappa \mathcal{F}_{\varkappa, \lambda}^{\aleph}\left(\zeta\left(\tau_{1}\right)-\zeta\left(\tau_{2}\right)\right)\right] \\
& \leq \xi\left(\zeta\left(\tau_{2}\right)\right)+\kappa \xi \mathcal{F}_{\varkappa, \lambda}^{\aleph}\left(\zeta\left(\tau_{1}\right)-\zeta\left(\tau_{2}\right)\right) \\
& =(\xi \circ \zeta)\left(\tau_{2}\right)+\kappa \mathcal{F}_{\varkappa, \lambda}^{\aleph}\left((\xi \circ \zeta)\left(\tau_{1}\right)-(\xi \circ \zeta)\left(\tau_{2}\right)\right),
\end{aligned}
$$

which completes the proof.

Theorem 7. Let $\zeta_{i}: \mathscr{H}=\left[\tau_{1}, \tau_{2}\right] \rightarrow \mathscr{R}$ be an arbitrary family of Raina-convex functions and let $\zeta(\tau)=\sup _{i} \zeta_{i}(\tau)$. If $\mathscr{H}=\left\{\tau \in\left[\tau_{1}, \tau_{2}\right]: \zeta(\tau)<+\infty\right\} \neq \emptyset$, then $\mathscr{H}$ is an interval and $\zeta$ is Raina-convex function.

Proof. $\forall \tau_{1}, \tau_{2} \in \mathscr{H}$ and $\kappa \in[0,1]$, we have

$$
\begin{aligned}
& \zeta\left(\kappa \tau_{1}+(1-\kappa) \tau_{2}\right) \\
& =\sup _{j} \zeta_{j}\left(\kappa \tau_{1}+(1-\kappa) \tau_{2}\right) \\
& \leq \sup _{j} \zeta_{j}\left(\tau_{2}\right)+\kappa \mathcal{F}_{\varkappa, \lambda}^{\aleph}\left(\sup _{j} \zeta_{j}\left(\tau_{1}\right)-\sup _{j} \zeta_{j}\left(\tau_{2}\right)\right) \\
& =\zeta\left(\tau_{2}\right)+\kappa \mathcal{F}_{\varkappa, \lambda}^{\aleph}\left(\zeta\left(\tau_{1}\right)-\zeta\left(\tau_{2}\right)\right)<+\infty,
\end{aligned}
$$

which completes the proof.

\section{New Version of H-H ANd FeJ́er-Type inequalities}

Theorem 8. Let $\zeta: \mathscr{H} \subseteq \mathscr{R} \rightarrow \mathscr{R}$ be a Raina-convex function with $\zeta \in L^{1}\left[\tau_{1} \cdot \tau_{2}\right]$, where $\tau_{1}, \tau_{2} \in \mathscr{H}$ with $\tau_{1}<\tau_{2}$, then

$\zeta\left(\frac{\tau_{1}+\tau_{2}}{2}\right)-\frac{1}{2\left(\tau_{2}-\tau_{1}\right)} \int_{\tau_{1}}^{\tau_{2}} \mathcal{F}_{\varkappa, \lambda}^{\aleph}\left(\zeta\left(\tau_{1}+\tau_{2}-\mu\right)-\zeta(\mu)\right) d \mu \leq \frac{1}{\tau_{2}-\tau_{1}} \int_{\tau_{1}}^{\tau_{2}} \zeta(\mu) d \mu$ 


$$
\leq \zeta\left(\tau_{2}\right)+\frac{1}{2} \mathcal{F}_{\varkappa, \lambda}^{\aleph}\left(\zeta\left(\tau_{1}\right)-\zeta\left(\tau_{2}\right)\right)
$$

Proof. Using (9), with $\mu=\kappa \tau_{1}+(1-\kappa) \tau_{2}, \nu=(1-\kappa) \tau_{1}+\kappa \tau_{2}$ and $\kappa=\frac{1}{2}$, we find that

$\zeta\left(\frac{\tau_{1}+\tau_{2}}{2}\right) \leq \zeta\left((1-\kappa) \tau_{1}+\kappa \tau_{2}\right)+\frac{1}{2} \mathcal{F}_{\varkappa, \lambda}^{\aleph}\left(\zeta\left(\kappa \tau_{1}+(1-\kappa) \tau_{2}\right)-\zeta\left((1-\kappa) \tau_{1}+\kappa \tau_{2}\right)\right)$

Thus by integrating, we obtain

$$
\begin{array}{r}
\zeta\left(\frac{\tau_{1}+\tau_{2}}{2}\right) \leq \int_{0}^{1} \zeta\left((1-\kappa) \tau_{1}+\kappa \tau_{2}\right) d \kappa+\frac{1}{2} \int_{0}^{1} \mathcal{F}_{\varkappa, \lambda}^{\aleph}\left(\zeta\left(\kappa \tau_{1}+(1-\kappa) \tau_{2}\right)\right. \\
\left.-\zeta\left((1-\kappa) \tau_{1}+\kappa \tau_{2}\right)\right) d \kappa \\
\leq \frac{1}{\tau_{2}-\tau_{1}} \int_{\tau_{1}}^{\tau_{2}} \zeta(\mu) d \mu+\frac{1}{2\left(\tau_{2}-\tau_{1}\right)} \int_{\tau_{1}}^{\tau_{2}} \mathcal{F}_{\varkappa, \lambda}^{\aleph}\left(\zeta\left(\tau_{1}+\tau_{2}-\mu\right)-\zeta(\mu)\right) d \mu
\end{array}
$$

So that

$\zeta\left(\frac{\tau_{1}+\tau_{2}}{2}\right)-\frac{1}{2\left(\tau_{2}-\tau_{1}\right)} \int_{\tau_{1}}^{\tau_{2}} \mathcal{F}_{\varkappa, \lambda}^{\aleph}\left(\zeta\left(\tau_{1}+\tau_{2}-\mu\right)-\zeta(\mu)\right) d \mu \leq \frac{1}{\tau_{2}-\tau_{1}} \int_{\tau_{1}}^{\tau_{2}} \zeta(\mu) d \mu$.

This completes the proof of left side of above inequality. For the right side using $\mu=\tau_{1}$ and $\nu=\tau_{2}$ in (9), and integrating over [0,1], we have

$$
\frac{1}{\tau_{2}-\tau_{1}} \int_{\tau_{1}}^{\tau_{2}} \zeta(\mu) d \mu \leq \zeta\left(\tau_{2}\right)+\frac{1}{2} \mathcal{F}_{\varkappa, \lambda}^{\aleph}\left(\zeta\left(\tau_{1}\right)-\zeta\left(\tau_{2}\right)\right)
$$

By simplification, the inequalities (11) and 12 , we get the inequality 100 .

Remark 1. Taking $\mathcal{F}_{\varkappa, \lambda}^{\aleph}(\mu-\nu)=\mu-\nu$, we reduce (10) to inequality 2).

Remark 2. Under the assumption of Theorem 8, if we take $\aleph=(1,1, \ldots)$ with $\varkappa=\alpha, \lambda=1$, we get the following inequality involving classical Mittag-Leffler function

$$
\begin{gathered}
\zeta\left(\frac{\tau_{1}+\tau_{2}}{2}\right)-\frac{1}{2\left(\tau_{2}-\tau_{1}\right)} \int_{\tau_{1}}^{\tau_{2}} E_{\alpha}\left(\zeta\left(\tau_{1}+\tau_{2}-\mu\right)-\zeta(\mu)\right) d \mu \leq \frac{1}{\tau_{2}-\tau_{1}} \int_{\tau_{1}}^{\tau_{2}} \zeta(\mu) d \mu \\
\leq \zeta\left(\tau_{2}\right)+\frac{1}{2} E_{\alpha}\left(\zeta\left(\tau_{1}\right)-\zeta\left(\tau_{2}\right)\right) .
\end{gathered}
$$

Theorem 9. Let $\zeta$ and $\xi$ be non-negative generalized convex functions of Raina type with $\zeta \xi \in L^{1}\left[\tau_{1}, \tau_{2}\right]$, where $\tau_{1}, \tau_{2} \in \mathscr{H}, \tau_{1}<\tau_{2}$. Then

$$
\frac{1}{\tau_{2}-\tau_{1}} \int_{\tau_{1}}^{\tau_{2}} \zeta(\mu) \xi(\mu) d \mu \leq M^{\prime}\left(\tau_{1}, \tau_{2}\right)
$$

where

$M^{\prime}\left(\tau_{1}, \tau_{2}\right)=\zeta\left(\tau_{2}\right) \xi\left(\tau_{2}\right)+\frac{1}{2} \zeta\left(\tau_{2}\right) \mathcal{F}_{\varkappa, \lambda}^{\aleph}\left(\xi\left(\tau_{1}\right)-\xi\left(\tau_{2}\right)\right)+\frac{1}{2} \xi\left(\tau_{2}\right) \mathcal{F}_{\varkappa, \lambda}^{\aleph}\left(\zeta\left(\tau_{1}\right)-\zeta\left(\tau_{2}\right)\right)$ 


$$
+\frac{1}{3} \mathcal{F}_{\varkappa, \lambda}^{\aleph}\left(\zeta\left(\tau_{1}\right)-\zeta\left(\tau_{2}\right)\right) \mathcal{F}_{\varkappa, \lambda}^{\aleph}\left(\xi\left(\tau_{1}\right)-\xi\left(\tau_{2}\right)\right)
$$

Proof. Since $\zeta$ and $\xi$ be a Raina-convex functions, we have

$$
\begin{aligned}
& \zeta\left(\kappa \tau_{1}+(1-\kappa) \tau_{2}\right) \leq \zeta\left(\tau_{2}\right)+\kappa \mathcal{F}_{\varkappa, \lambda}^{\aleph}\left(\zeta\left(\tau_{1}\right)-\zeta\left(\tau_{2}\right)\right) \\
& \xi\left(\kappa \tau_{1}+(1-\kappa) \tau_{2}\right) \leq \xi\left(\tau_{2}\right)+\kappa \mathcal{F}_{\varkappa, \lambda}^{\aleph}\left(\xi\left(\tau_{1}\right)-\xi\left(\tau_{2}\right)\right)
\end{aligned}
$$

For all $\kappa \in[0,1]$. Since $\zeta$ and $\xi$ are non-negative, we have

$$
\begin{array}{r}
\zeta\left(\kappa \tau_{1}+(1-\kappa) \tau_{2}\right) \xi\left(\kappa \tau_{1}+(1-\kappa) \tau_{2}\right) \leq \zeta\left(\tau_{2}\right) \xi\left(\tau_{2}\right)+\kappa \zeta\left(\tau_{2}\right) \mathcal{F}_{\varkappa, \lambda}^{\aleph}\left(\xi\left(\tau_{1}\right)-\xi\left(\tau_{2}\right)\right) \\
+\kappa \xi\left(\tau_{2}\right) \mathcal{F}_{\varkappa, \lambda}^{\aleph}\left(\zeta\left(\tau_{1}\right)-\zeta\left(\tau_{2}\right)\right)+\kappa^{2} \mathcal{F}_{\varkappa, \lambda}^{\aleph}\left(\zeta\left(\tau_{1}\right)-\zeta\left(\tau_{2}\right)\right) \mathcal{F}_{\varkappa, \lambda}^{\aleph}\left(\xi\left(\tau_{1}\right)-\xi\left(\tau_{2}\right)\right)
\end{array}
$$

integrating over $[0,1]$ both sides, we have

$$
\begin{aligned}
& \int_{0}^{1} \zeta\left(\kappa \tau_{1}+(1-\kappa) \tau_{2}\right) \xi\left(\kappa \tau_{1}+(1-\kappa) \tau_{2}\right) d \kappa \leq \zeta\left(\tau_{2}\right) \xi\left(\tau_{2}\right) \\
& +\frac{1}{2} \zeta\left(\tau_{2}\right) \mathcal{F}_{\varkappa, \lambda}^{\aleph}\left(\xi\left(\tau_{1}\right)-\xi\left(\tau_{2}\right)\right)+\frac{1}{2} \xi\left(\tau_{2}\right) \mathcal{F}_{\varkappa, \lambda}^{\aleph}\left(\zeta\left(\tau_{1}\right)-\zeta\left(\tau_{2}\right)\right) \\
& \left.+\frac{1}{3} \mathcal{F}_{\varkappa, \lambda}^{\aleph}\left(\zeta\left(\tau_{1}\right)-\zeta \tau_{2}\right)\right) \mathcal{F}_{\varkappa, \lambda}^{\aleph}\left(\xi\left(\tau_{1}\right)-\xi\left(\tau_{2}\right)\right)
\end{aligned}
$$

then

$$
\frac{1}{\tau_{2}-\tau_{1}} \int_{\tau_{1}}^{\tau_{2}} \zeta(\mu) \xi(\mu) d \mu \leq M^{\prime}\left(\tau_{1}, \tau_{2}\right)
$$

Remark 3. Taking $\mathcal{F}_{\varkappa, \lambda}^{\aleph}(\mu-\nu)=\mu-\nu$ in above inequality (14), we get inequality (1.4) in [13].

Remark 4. Under the assumption of Theorem 9, if we take $\aleph=(1,1, \ldots)$ with $\varkappa=\alpha, \lambda=1$, we get the following inequality involving classical Mittag-Leffler function

$$
\frac{1}{\tau_{2}-\tau_{1}} \int_{\tau_{1}}^{\tau_{2}} \zeta(\mu) \xi(\mu) d \mu \leq M^{\prime}\left(\tau_{1}, \tau_{2}\right)
$$

where

$$
\begin{array}{r}
M^{\prime}\left(\tau_{1}, \tau_{2}\right)=\zeta\left(\tau_{2}\right) \xi\left(\tau_{2}\right)+\frac{1}{2} \zeta\left(\tau_{2}\right) E_{\alpha}\left(\xi\left(\tau_{1}\right)-\xi\left(\tau_{2}\right)\right)+\frac{1}{2} \xi\left(\tau_{2}\right) E_{\alpha}\left(\zeta\left(\tau_{1}\right)-\zeta\left(\tau_{2}\right)\right) \\
+\frac{1}{3} E_{\alpha}\left(\zeta\left(\tau_{1}\right)-\zeta\left(\tau_{2}\right)\right) E_{\alpha}\left(\xi\left(\tau_{1}\right)-\xi\left(\tau_{2}\right)\right)
\end{array}
$$

Theorem 10. Let $\zeta$ be a Raina-convex function with $\zeta \in L^{1}\left[\tau_{1}, \tau_{2}\right]$, where $\tau_{1}, \tau_{2} \in$ $\mathscr{H}, \tau_{1}<\tau_{2}$, and $\xi: \mathscr{H}=\left[\tau_{1}, \tau_{2}\right] \rightarrow \mathscr{R}$ be non-negative, integrable symmetric about $\frac{\tau_{1}+\tau_{2}}{2}$, then

$$
\int_{\tau_{1}}^{\tau_{2}} \zeta(\mu) \xi(\mu) d \mu \leq\left[\zeta\left(\tau_{2}\right)+\frac{1}{2} \mathcal{F}_{\varkappa, \lambda}^{\aleph}\left(\zeta\left(\tau_{1}\right)-\zeta\left(\tau_{2}\right)\right)\right] \int_{\tau_{1}}^{\tau_{2}} \xi(\mu) d \mu .
$$


Proof. Since $\zeta$ be a Raina-convex function and $\xi$ is non-negative integrable and symmetric about $\frac{\tau_{1}+\tau_{2}}{2}$, we find that

$$
\begin{aligned}
& \int_{\tau_{1}}^{\tau_{2}} \zeta(\mu) \xi(\mu) d \mu=\frac{1}{2}\left[\int_{\tau_{1}}^{\tau_{2}} \zeta(\mu) \xi(\mu) d \mu+\int_{\tau_{1}}^{\tau_{2}} \zeta\left(\tau_{1}+\tau_{2}-\mu\right) g\left(\tau_{1}+\tau_{2}-\mu\right) d \mu\right] \\
& =\frac{1}{2} \int_{\tau_{1}}^{\tau_{2}}\left[\left(\zeta(\mu)+\zeta\left(\tau_{1}+\tau_{2}-\mu\right)\right) \xi(\mu) d \mu\right] \\
& =\frac{1}{2} \int_{\tau_{1}}^{\tau_{2}}\left[\zeta\left(\frac{\tau_{2}-\mu}{\tau_{2}-\tau_{1}} \tau_{1}+\frac{\mu-\tau_{1}}{\tau_{2}-\tau_{1}} \tau_{2}\right)+\zeta\left(\frac{\mu-\tau_{1}}{\tau_{2}-\tau_{1}} \tau_{1}+\frac{\tau_{2}-\mu}{\tau_{2}-\tau_{1}} \tau_{2}\right)\right] \xi(\mu) d \mu \\
& \leq \frac{1}{2} \int_{\tau_{1}}^{\tau_{2}}\left[\left(\zeta\left(\tau_{2}\right)+\frac{\tau_{2}-\mu}{\tau_{2}-\tau_{1}} \mathcal{F}_{\varkappa, \lambda}^{\aleph}\left(\zeta\left(\tau_{1}\right)-\zeta\left(\tau_{2}\right)\right)\right)\right. \\
& \left.+\left(\zeta\left(\tau_{2}\right)+\frac{\mu-\tau_{1}}{\tau_{2}-\tau_{1}} \mathcal{F}_{\varkappa, \lambda}^{\aleph}\left(\zeta\left(\tau_{1}\right)-\zeta\left(\tau_{2}\right)\right)\right)\right] \xi(\mu) d \mu \\
& \leq\left[\zeta\left(\tau_{2}\right)+\frac{1}{2} \mathcal{F}_{\varkappa, \lambda}^{\aleph}\left(\zeta\left(\tau_{1}\right)-\zeta\left(\tau_{2}\right)\right)\right] \int_{\tau_{1}}^{\tau_{2}} \xi(\mu) d \mu,
\end{aligned}
$$

which completes the proof.

Remark 5. (i) Taking $\mathcal{F}_{\varkappa, \lambda}^{\aleph}(\mu-\nu)=\mu-\nu$ and $\xi(x)=1$, then inequality (16) reduce to the inequality (2).

(ii) Taking $\mathcal{F}_{\varkappa, \lambda}^{\aleph}(\mu-\nu)=\mu-\nu$, then inequality (16) reduce to the inequality (3).

(iii) Under the assumption of Theorem 10 , if we take $\aleph=(1,1, \ldots)$ with $\varkappa=\alpha, \lambda=$ 1, we get the following inequality involving classical Mittag-Leffler function

$$
\int_{\tau_{1}}^{\tau_{2}} \zeta(\mu) \xi(\mu) d \mu \leq\left[\zeta\left(\tau_{2}\right)+\frac{1}{2} E_{\alpha}\left(\zeta\left(\tau_{1}\right)-\zeta\left(\tau_{2}\right)\right)\right] \int_{\tau_{1}}^{\tau_{2}} \xi(\mu) d \mu .
$$

\section{NeW integral type inequalities Via Raina-CONVEx function}

Theorem 11. Suppose $\zeta: \mathscr{H} \subseteq \mathscr{R} \rightarrow \mathscr{R}$ be a diff mapp on $\mathscr{H}^{\circ}$ with $\zeta^{\prime} \in L^{1}\left[\tau_{1}, \tau_{2}\right]$, where $\tau_{1}, \tau_{2} \in \mathscr{H}, \tau_{1}<\tau_{2}$. If $\left|\zeta^{\prime}(\mu)\right|^{\ell}$ for $\ell \geq 1$ is Raina-convex function on $\left[\tau_{1}, \tau_{2}\right]$ and $0 \leq \alpha, \beta \leq 1$ then

$$
\begin{aligned}
& \left|\frac{\alpha \zeta\left(\tau_{1}\right)+\beta \zeta\left(\tau_{2}\right)}{2}+\frac{2-\alpha-\beta}{2} \zeta\left(\frac{\tau_{1}+\tau_{2}}{2}\right)-\frac{1}{\tau_{2}-\tau_{1}} \int_{\tau_{1}}^{\tau_{2}} \zeta(\mu) d \mu\right| \\
& \leq \frac{\tau_{2}-\tau_{1}}{8}\left(\frac{1}{6}\right)^{\frac{1}{\ell}}\left\{( 1 - 2 \alpha + 2 \alpha ^ { 2 } ) ^ { 1 - \frac { 1 } { \ell } } \left[\left(6-12 \alpha+12 \alpha^{2}\right)\left|\zeta^{\prime}\left(\tau_{2}\right)\right|^{\ell}+\left(4-9 \alpha+12 \alpha^{2}-2 \alpha^{3}\right)\right.\right. \\
& \left.\times \mathcal{F}_{\varkappa, \lambda}^{\aleph}\left(\left|\zeta^{\prime}\left(\tau_{1}\right)\right|^{\ell}-\left|\zeta^{\prime}\left(\tau_{2}\right)\right|^{\ell}\right)\right]^{\frac{1}{\ell}}+\left(1-2 \beta+2 \beta^{2}\right)^{1-\frac{1}{\ell}}\left[\left(6-12 \beta+12 \beta^{2}\right)\left|\zeta^{\prime}\left(\tau_{2}\right)\right|^{\ell}\right.
\end{aligned}
$$


$\left.\left.+\left(2-3 \beta+2 \beta^{3}\right) \mathcal{F}_{\varkappa, \lambda}^{\aleph}\left(\left|\zeta^{\prime}\left(\tau_{1}\right)\right|^{\ell}-\left|\zeta^{\prime}\left(\tau_{2}\right)\right|^{\ell}\right)\right]^{\frac{1}{\ell}}\right\}$.

Proof. In case $\ell>1$, using lemma (1), Raina-convexity of $\left|\zeta^{\prime}(x)\right|^{\ell}$ on $\left[\tau_{1}, \tau_{2}\right]$ and power mean inequality, we have

$$
\begin{aligned}
& \left|\frac{\alpha \zeta\left(\tau_{1}\right)+\beta \zeta\left(\tau_{2}\right)}{2}+\frac{2-\alpha-\beta}{2} \zeta\left(\frac{\tau_{1}+\tau_{2}}{2}\right)-\frac{1}{\tau_{2}-\tau_{1}} \int_{\tau_{1}}^{\tau_{2}} \zeta(\mu) d \mu\right| \\
& \leq \frac{\tau_{2}-\tau_{1}}{4}\left[\int_{0}^{1}|1-\alpha-\kappa|\left|\zeta^{\prime}\left(\kappa \tau_{1}+(1-\kappa) \frac{\tau_{1}+\tau_{2}}{2}\right)\right| d \kappa\right. \\
& \left.+\int_{0}^{1}|\beta-\kappa|\left|\zeta^{\prime}\left(\kappa \frac{\tau_{1}+\tau_{2}}{2}+(1-\kappa) \tau_{2}\right)\right| d \kappa\right] \\
& \leq \frac{\tau_{2}-\tau_{1}}{4}\left[( \int _ { 0 } ^ { 1 } | 1 - \alpha - \kappa | d \kappa ) ^ { 1 - \frac { 1 } { \ell } } \left[\int _ { 0 } ^ { 1 } | 1 - \alpha - \kappa | \left(\left|\zeta^{\prime}\left(\tau_{2}\right)\right|^{\ell}+\left(\frac{1+\kappa}{2}\right)\right.\right.\right. \\
& \left.\left.\times \mathcal{F}_{\varkappa, \lambda}^{\aleph}\left(\left|\zeta^{\prime}\left(\tau_{1}\right)\right|^{\ell}-\left|\zeta^{\prime}\left(\tau_{2}\right)\right|^{\ell}\right)\right) d \kappa\right]^{\frac{1}{\ell}}+\left(\int_{0}^{1}|\beta-\kappa| d \kappa\right)^{1-\frac{1}{\ell}} \\
& \left.\times\left[\int_{0}^{1}|\beta-\kappa|\left(\left|\zeta^{\prime}\left(\tau_{2}\right)\right|^{\ell}+\frac{\kappa}{2} \mathcal{F}_{\varkappa, \lambda}^{\aleph}\left(\left|\zeta^{\prime}\left(\tau_{1}\right)\right|^{\ell}-\left|\zeta^{\prime}\left(\tau_{2}\right)\right|^{\ell}\right)\right) d \kappa\right]^{\frac{1}{\ell}}\right]
\end{aligned}
$$

using lemma (2), by simplifications we obtain

$$
\begin{aligned}
& \int_{0}^{1}|1-\alpha-\kappa|\left(\left|\zeta^{\prime}\left(\tau_{2}\right)\right|^{\ell}+\left(\frac{1+\kappa}{2}\right) \mathcal{F}_{\varkappa, \lambda}^{\aleph}\left(\left|\zeta^{\prime}\left(\tau_{1}\right)\right|^{\ell}-\left|\zeta^{\prime}\left(\tau_{2}\right)\right|^{\ell}\right)\right) d \kappa \\
& =\left(\left|\zeta^{\prime}\left(\tau_{2}\right)\right|^{\ell}+\frac{1}{2} \mathcal{F}_{\varkappa, \lambda}^{\aleph}\left(\left|\zeta^{\prime}\left(\tau_{1}\right)\right|^{\ell}-\left|\zeta^{\prime}\left(\tau_{2}\right)\right|^{\ell}\right)\right) \int_{0}^{1}|1-\alpha-\kappa| d \kappa \\
& +\frac{1}{2} \mathcal{F}_{\varkappa, \lambda}^{\aleph}\left(\left|\zeta^{\prime}\left(\tau_{1}\right)\right|^{\ell}-\left|\zeta^{\prime}\left(\tau_{2}\right)\right|^{\ell}\right) \int_{0}^{1} \kappa|1-\alpha-\kappa| d \kappa \\
& =\left(\left|\zeta^{\prime}\left(\tau_{2}\right)\right|^{\ell}+\frac{1}{2} \mathcal{F}_{\varkappa, \lambda}^{\aleph}\left(\left|\zeta^{\prime}\left(\tau_{1}\right)\right|^{\ell}-\left|\zeta^{\prime}\left(\tau_{2}\right)\right|^{\ell}\right)\right)\left(\frac{1}{2}-\alpha+\alpha^{2}\right) \\
& +\frac{1}{12} \mathcal{F}_{\varkappa, \lambda}^{\aleph}\left(\left|\zeta^{\prime}\left(\tau_{1}\right)\right|^{\ell}-\left|\zeta^{\prime}\left(\tau_{2}\right)\right|^{\ell}\right)\left[(1-\alpha)^{3}+\alpha^{2}(3-\alpha)\right] \\
& =\frac{1}{2}\left(1-2 \alpha+2 \alpha^{2}\right)\left|\zeta^{\prime}\left(\tau_{2}\right)\right|^{\ell}+\frac{1}{12}\left(4-9 \alpha+12 \alpha^{2}-2 \alpha^{3}\right) \mathcal{F}_{\varkappa, \lambda}^{\aleph}\left(\left|\zeta^{\prime}\left(\tau_{1}\right)\right|^{\ell}-\left|\zeta^{\prime}\left(\tau_{2}\right)\right|^{\ell}\right)
\end{aligned}
$$

and

$$
\begin{aligned}
& \int_{0}^{1}|\beta-\kappa|\left(\left|\zeta^{\prime}\left(\tau_{2}\right)\right|^{\ell}+\left(\frac{\kappa}{2}\right) \mathcal{F}_{\varkappa, \lambda}^{\aleph}\left(\left|\zeta^{\prime}\left(\tau_{1}\right)\right|^{\ell}-\left|\zeta^{\prime}\left(\tau_{2}\right)\right|^{\ell}\right)\right) d \kappa \\
& =\left|\zeta^{\prime}\left(\tau_{2}\right)\right|^{\ell} \int_{0}^{1}|\beta-t| d \kappa+\frac{1}{2} \mathcal{F}_{\varkappa, \lambda}^{\aleph}\left(\left|\zeta^{\prime}\left(\tau_{1}\right)\right|^{\ell}-\left|\zeta^{\prime}\left(\tau_{2}\right)\right|^{\ell}\right) \int_{0}^{1} \kappa|\beta-\kappa| d \kappa
\end{aligned}
$$




$$
\begin{aligned}
& =\left|\zeta^{\prime}\left(\tau_{2}\right)\right|^{\ell}\left(\frac{1}{2}-\beta-\beta^{2}\right)+\frac{1}{12} \mathcal{F}_{\varkappa, \lambda}^{\aleph}\left(\left|\zeta^{\prime}\left(\tau_{1}\right)\right|^{\ell}-\left|\zeta^{\prime}\left(\tau_{2}\right)\right|^{\ell}\right)\left(\beta^{3}+(2+\beta)(1-\beta)^{2}\right) \\
& =\left.\frac{1}{2}\left(1-2 \beta+2 \beta^{2}\right) \zeta^{\prime}\left(\tau_{2}\right)\right|^{\ell}+\frac{1}{12}\left(2-3 \beta+2 \beta^{3}\right) \mathcal{F}_{\varkappa, \lambda}^{\aleph}\left(\left|\zeta^{\prime}\left(\tau_{1}\right)\right|^{\ell}-\left|\zeta^{\prime}\left(\tau_{2}\right)\right|^{\ell}\right),
\end{aligned}
$$

The following above two inequalities substitute into inequality $(19)$ and according lemma (2) result in inequality $(18)$ for $\ell>1$.

For $\ell=1$, from lemma (1) and (2) it follows that

$$
\begin{aligned}
& \left|\frac{\alpha \zeta\left(\tau_{1}\right)+\beta \zeta\left(\tau_{2}\right)}{2}+\frac{2-\alpha-\beta}{2} \zeta\left(\frac{\tau_{1}+\tau_{2}}{2}\right)-\frac{1}{\tau_{2}-\tau_{1}} \int_{\tau_{1}}^{\tau_{2}} \zeta(\mu) d \mu\right| \\
& \leq \frac{\tau_{2}-\tau_{1}}{4}\left[\int_{0}^{1}|1-\alpha-\kappa|\left(\left|\zeta^{\prime}\left(\tau_{2}\right)\right|+\left(\frac{1+\kappa}{2}\right) \mathcal{F}_{\varkappa, \lambda}^{\aleph}\left(\left|\zeta^{\prime}\left(\tau_{1}\right)\right|-\left|\zeta^{\prime}\left(\tau_{2}\right)\right|\right)\right) d \kappa\right. \\
& \left.+\int_{0}^{1}|\beta-\kappa|\left(\left|\zeta^{\prime}\left(\tau_{2}\right)\right|+\frac{\kappa}{2} \mathcal{F}_{\varkappa, \lambda}^{\aleph}\left(\left|\zeta^{\prime}\left(\tau_{1}\right)\right|-\left|\zeta^{\prime}\left(\tau_{2}\right)\right|\right)\right) d \kappa\right] \\
& =\frac{\tau_{2}-\tau_{1}}{48}\left\{\left(6-12 \alpha+12 \alpha^{2}\right)\left|\zeta^{\prime}\left(\tau_{2}\right)\right|+\left(4-9 \alpha+12 \alpha^{2}-2 \alpha^{3}\right) \mathcal{F}_{\varkappa, \lambda}^{\aleph}\left(\left|\zeta^{\prime}\left(\tau_{1}\right)\right|-\left|\zeta^{\prime}\left(\tau_{2}\right)\right|\right)\right. \\
& \left.+\left(6-12 \beta+12 \beta^{2}\right)\left|\zeta^{\prime}\left(\tau_{2}\right)\right|+\left(2-3 \beta+2 \beta^{3}\right) \mathcal{F}_{\varkappa, \lambda}^{\aleph}\left(\left|\zeta^{\prime}\left(\tau_{1}\right)\right|-\left|\zeta^{\prime}\left(\tau_{2}\right)\right|\right)\right\} .
\end{aligned}
$$

Remark 6. $(i)$ Choosing $\mathcal{F}_{\varkappa, \lambda}^{\aleph}(\mu-\nu)=(\mu-\nu)$, then the inequality 18 collapse to the inequality (3.1) in [17].

(ii) Under the assumption of Theorem 11 , if we take $\aleph=(1,1, \ldots)$ with $\varkappa=\alpha, \lambda=$ 1, we get the following inequality involving classical Mittag-Leffler function

$$
\begin{aligned}
& \left|\frac{\alpha \zeta\left(\tau_{1}\right)+\beta \zeta\left(\tau_{2}\right)}{2}+\frac{2-\alpha-\beta}{2} \zeta\left(\frac{\tau_{1}+\tau_{2}}{2}\right)-\frac{1}{\tau_{2}-\tau_{1}} \int_{\tau_{1}}^{\tau_{2}} \zeta(\mu) d \mu\right| \leq \frac{\tau_{2}-\tau_{1}}{8} \\
& \times\left(\frac{1}{6}\right)^{\frac{1}{\ell}}\left\{( 1 - 2 \alpha + 2 \alpha ^ { 2 } ) ^ { 1 - \frac { 1 } { \ell } } \left[\left(6-12 \alpha+12 \alpha^{2}\right)\left|\zeta^{\prime}\left(\tau_{2}\right)\right|^{\ell}+\left(4-9 \alpha+12 \alpha^{2}-2 \alpha^{3}\right)\right.\right. \\
& \left.\times E_{\alpha}\left(\left|\zeta^{\prime}\left(\tau_{1}\right)\right|^{\ell}-\left|\zeta^{\prime}\left(\tau_{2}\right)\right|^{\ell}\right)\right]^{\frac{1}{\ell}}+\left(1-2 \beta+2 \beta^{2}\right)^{1-\frac{1}{\ell}}\left[\left(6-12 \beta+12 \beta^{2}\right)\left|\zeta^{\prime}\left(\tau_{2}\right)\right|^{\ell}\right. \\
& \left.\left.+\left(2-3 \beta+2 \beta^{3}\right) E_{\alpha}\left(\left|\zeta^{\prime}\left(\tau_{1}\right)\right|^{\ell}-\left|\zeta^{\prime}\left(\tau_{2}\right)\right|^{\ell}\right)\right]^{\frac{1}{\ell}}\right\}
\end{aligned}
$$

(iii) Choosing $\alpha=\beta$ in above Theorem (11), we derive the following corollary,

Corollary 1. Let $\zeta: \mathscr{H} \subseteq \mathscr{R} \rightarrow \mathscr{R}$ be a diff mapp on $\mathscr{H}^{\circ}$ with $\zeta^{\prime} \in L_{1}\left[\tau_{1}, \tau_{2}\right]$, where $\tau_{1}, \tau_{2} \in \mathscr{H}, \tau_{1}<\tau_{2}$. If $\left|\zeta^{\prime}(\mu)\right|^{\ell}$ for $\ell \geq 1$ is Raina-convex function on 
$\left[\tau_{1}, \tau_{2}\right]$ and $0 \leq \alpha \leq 1$ then

$$
\begin{aligned}
& \left|\frac{\alpha}{2}\left[\zeta\left(\tau_{1}\right)+\zeta\left(\tau_{2}\right)\right]+(1-\alpha) \zeta\left(\frac{\tau_{1}+\tau_{2}}{2}\right)-\frac{1}{\tau_{1}-\tau_{2}} \zeta(\mu) d \mu\right| \leq \frac{\tau_{2}-\tau_{1}}{8}\left(\frac{1}{6}\right)^{\frac{1}{\ell}} \\
& \times\left\{( 1 - 2 \alpha + 2 \alpha ^ { 2 } ) ^ { 1 - \frac { 1 } { \ell } } \left[\left(6-12 \alpha+12 \alpha^{2}\right)\left|\zeta^{\prime}\left(\tau_{2}\right)\right|^{\ell}\right.\right. \\
& \left.+\left(4-9 \alpha+12 \alpha^{2}-2 \alpha^{3}\right) \mathcal{F}_{\varkappa, \lambda}^{\aleph}\left(\left|\zeta^{\prime}\left(\tau_{1}\right)\right|^{\ell}\right)-\left|\zeta^{\prime}\left(\tau_{2}\right)\right|^{\ell}\right]^{\frac{1}{\ell}} \\
& \left.+\left[\left(6-12 \alpha+12 \alpha^{2}\right)\left|\zeta^{\prime}\left(\tau_{2}\right)\right|^{\ell}+\left(2-3 \alpha+2 \alpha^{3}\right) \mathcal{F}_{\varkappa, \lambda}^{\aleph}\left(\left|\zeta^{\prime}\left(\tau_{1}\right)\right|^{\ell}-\left|\zeta^{\prime}\left(\tau_{2}\right)\right|^{\ell}\right)\right]^{\frac{1}{\ell}}\right\} .
\end{aligned}
$$

Remark 7. (i) Choosing $\mathcal{F}_{\varkappa, \lambda}^{\aleph}(\mu-\nu)=\mu-\nu$ in Corollary (1), then inequality (22) collapse to inequality (3.5) in [17].

(ii) Under the assumption of Corollary 1 , if we take $\aleph=(1,1, \ldots)$ with $\varkappa=\alpha, \lambda=$ 1, we get the following inequality involving classical Mittag-Leffler function

$$
\begin{aligned}
& \left|\frac{\alpha}{2}\left[\zeta\left(\tau_{1}\right)+\zeta\left(\tau_{2}\right)\right]+(1-\alpha) \zeta\left(\frac{\tau_{1}+\tau_{2}}{2}\right)-\frac{1}{\tau_{1}-\tau_{2}} \zeta(\mu) d \mu\right| \leq \frac{\tau_{2}-\tau_{1}}{8}\left(\frac{1}{6}\right)^{\frac{1}{\ell}} \\
& \times\left[( 1 - 2 \alpha + 2 \alpha ^ { 2 } ) ^ { 1 - \frac { 1 } { \ell } } \left[\left(6-12 \alpha+12 \alpha^{2}\right)\left|\zeta^{\prime}\left(\tau_{2}\right)\right|^{\ell}\right.\right. \\
& \left.+\left(4-9 \alpha+12 \alpha^{2}-2 \alpha^{3}\right) E_{\alpha}\left(\left|\zeta^{\prime}\left(\tau_{1}\right)\right|^{\ell}\right)-\left|\zeta^{\prime}\left(\tau_{2}\right)\right|^{\ell}\right]^{\frac{1}{\ell}} \\
& \left.+\left[\left(6-12 \alpha+12 \alpha^{2}\right)\left|\zeta^{\prime}\left(\tau_{2}\right)\right|^{\ell}+\left(2-3 \alpha+2 \alpha^{3}\right) E_{\alpha}\left(\left|\zeta^{\prime}\left(\tau_{1}\right)\right|^{\ell}-\left|\zeta^{\prime}\left(\tau_{2}\right)\right|^{\ell}\right)\right]^{\frac{1}{\ell}}\right] .
\end{aligned}
$$

(iii) choosing $\alpha=\beta=\frac{1}{2}, \frac{1}{3}$, respectively, in above Theorem (11), we can obtain the following inequality,

Corollary 2. Suppose $\zeta: \mathscr{H} \subseteq \mathscr{R} \rightarrow \mathscr{R}$ be a diff mapp on $\mathscr{H}^{\circ}$ with $\zeta^{\prime} \in L^{1}\left[\tau_{1}, \tau_{2}\right]$ where $\tau_{1}, \tau_{2} \in \mathscr{H}, \tau_{1}<\tau_{2}$. If $\left|\zeta^{\prime}(\mu)\right|^{\ell}$ for $\ell \geq 1$ is Raina-convex function on $\left[\tau_{1}, \tau_{2}\right]$ and $0 \leq \alpha, \beta \leq 1$, then

$$
\begin{aligned}
& \left|\frac{1}{2}\left[\frac{\zeta\left(\tau_{1}\right)+\zeta\left(\tau_{2}\right)}{2}+\zeta\left(\frac{\tau_{1}+\tau_{2}}{2}\right)\right]-\frac{1}{\tau_{2}-\tau_{1}} \int_{\tau_{1}}^{\tau_{2}} \zeta(x) d x\right| \leq \frac{\tau_{2}-\tau_{1}}{16}\left(\frac{1}{12}\right)^{\frac{1}{\ell}} \\
& \times\left\{\left[12\left|\zeta^{\prime}\left(\tau_{2}\right)\right|^{\ell}+9 \mathcal{F}_{\varkappa, \lambda}^{\aleph}\left(\left|\zeta^{\prime}\left(\tau_{1}\right)\right|^{\ell}-\left|\zeta^{\prime}\left(\tau_{2}\right)\right|^{\ell}\right)\right]^{\frac{1}{\ell}}\right. \\
& \left.+\left[12\left|\zeta^{\prime}\left(\tau_{2}\right)\right|^{\ell}+3 \mathcal{F}_{\varkappa, \lambda}^{\aleph}\left(\left|\zeta^{\prime}\left(\tau_{1}\right)\right|^{\ell}-\left|\zeta^{\prime}\left(\tau_{2}\right)\right|^{\ell}\right)\right]^{\frac{1}{\ell}}\right\}
\end{aligned}
$$




$$
\begin{aligned}
& \left|\frac{1}{6}\left[\zeta\left(\tau_{1}\right)+\zeta\left(\tau_{2}\right)+4 \zeta\left(\frac{\tau_{1}+\tau_{2}}{2}\right)\right]-\frac{1}{\tau_{2}-\tau_{1}} \int_{\tau_{1}}^{\tau_{2}} \zeta(x) d x\right| \\
& \leq \frac{5\left(\tau_{2}-\tau_{1}\right)}{72}\left(\frac{1}{90}\right)^{\frac{1}{\ell}}\left\{\left[90\left|\zeta^{\prime}\left(\tau_{2}\right)\right|^{\ell}+61 \mathcal{F}_{\varkappa, \lambda}^{\aleph}\left(\left|\zeta^{\prime}\left(\tau_{1}\right)\right|^{\ell}-\left|\zeta^{\prime}\left(\tau_{2}\right)\right|^{\ell}\right)\right]^{\frac{1}{\ell}}\right. \\
& \left.+\left[90\left|\zeta^{\prime}\left(\tau_{2}\right)\right|^{\ell}+29 \mathcal{F}_{\varkappa, \lambda}^{\aleph}\left(\left|\zeta^{\prime}\left(\tau_{1}\right)\right|^{\ell}-\left|\zeta^{\prime}\left(\tau_{2}\right)\right|^{\ell}\right)\right]^{\frac{1}{\ell}}\right\} .
\end{aligned}
$$

Remark 8. Under the assumption of Corollary 2, if we take $\aleph=(1,1, \ldots)$ with $\varkappa=\alpha, \lambda=1$, we get the following inequality involving classical Mittag-Leffler function

$$
\begin{aligned}
& \left|\frac{1}{2}\left[\frac{\zeta\left(\tau_{1}\right)+\zeta\left(\tau_{2}\right)}{2}+\zeta\left(\frac{\tau_{1}+\tau_{2}}{2}\right)\right]-\frac{1}{\tau_{2}-\tau_{1}} \int_{\tau_{1}}^{\tau_{2}} \zeta(\mu) d \mu\right| \leq \frac{\tau_{2}-\tau_{1}}{16}\left(\frac{1}{12}\right)^{\frac{1}{\ell}} \\
& \times\left\{\left[12\left|\zeta^{\prime}\left(\tau_{2}\right)\right|^{\ell}+9 E_{\alpha}\left(\left|\zeta^{\prime}\left(\tau_{1}\right)\right|^{\ell}-\left|\zeta^{\prime}\left(\tau_{2}\right)\right|^{\ell}\right)\right]^{\frac{1}{\ell}}\right. \\
& \left.+\left[12\left|\zeta^{\prime}\left(\tau_{2}\right)\right|^{\ell}+3 E_{\alpha}\left(\left|\zeta^{\prime}\left(\tau_{1}\right)\right|^{\ell}-\left|\zeta^{\prime}\left(\tau_{2}\right)\right|^{\ell}\right)\right]^{\frac{1}{\ell}}\right\} \\
& \left|\frac{1}{6}\left[\zeta\left(\tau_{1}\right)+\zeta\left(\tau_{2}\right)+4 \zeta\left(\frac{\tau_{1}+\tau_{2}}{2}\right)\right]-\frac{1}{\tau_{2}-\tau_{1}} \int_{\tau_{1}}^{\tau_{2}} \zeta(\mu) d \mu\right| \\
& \leq \frac{5\left(\tau_{2}-\tau_{1}\right)}{72}\left(\frac{1}{90}\right)^{\frac{1}{\ell}}\left\{\left[90\left|\zeta^{\prime}\left(\tau_{2}\right)\right|^{\ell}+61 E_{\alpha}\left(\left|\zeta^{\prime}\left(\tau_{1}\right)\right|^{\ell}-\left|\zeta^{\prime}\left(\tau_{2}\right)\right|^{\ell}\right)\right]^{\frac{1}{\ell}}\right. \\
& \left.+\left[90\left|\zeta^{\prime}\left(\tau_{2}\right)\right|^{\ell}+29 E_{\alpha}\left(\left|\zeta^{\prime}\left(\tau_{1}\right)\right|^{\ell}-\left|\zeta^{\prime}\left(\tau_{2}\right)\right|^{\ell}\right)\right]^{\frac{1}{\ell}}\right\} .
\end{aligned}
$$

If we choose $\ell=1$ in Corollary (2), then we take the following inequality,

Corollary 3. Suppose $\zeta: \mathscr{H} \subseteq \mathscr{R} \rightarrow \mathscr{R}$ be a diff mapp on $\mathscr{H}^{\circ}$ with $\zeta^{\prime} \in L^{1}\left[\tau_{1}, \tau_{2}\right]$ where $\tau_{1}, \tau_{2} \in \mathscr{H}, \tau_{1}<\tau_{2}$. If $\left|\zeta^{\prime}(\mu)\right|$ is Raina-convex function on $\left[\tau_{1}, \tau_{2}\right]$

$$
\begin{aligned}
& \left|\frac{1}{2}\left[\frac{\zeta\left(\tau_{1}\right)+\zeta\left(\tau_{2}\right)}{2}+\zeta\left(\frac{\tau_{1}+\tau_{2}}{2}\right)\right]-\frac{1}{\tau_{2}-\tau_{1}} \int_{\tau_{1}}^{\tau_{2}} \zeta(\mu) d \mu\right| \\
& \leq \frac{\tau_{2}-\tau_{1}}{16}\left[2\left|\zeta^{\prime}\left(\tau_{2}\right)\right|+\mathcal{F}_{\varkappa, \lambda}^{\aleph}\left(\left|\zeta^{\prime}\left(\tau_{1}\right)\right|-\left|\zeta^{\prime}\left(\tau_{2}\right)\right|\right)\right] \\
& \left|\frac{1}{6}\left[\zeta\left(\tau_{1}\right)+\zeta\left(\tau_{2}\right)+4 \zeta\left(\frac{\tau_{1}+\tau_{2}}{2}\right)\right]-\frac{1}{\tau_{2}-\tau_{1}} \int_{\tau_{1}}^{\tau_{2}} \zeta(\mu) d \mu\right|
\end{aligned}
$$




$$
\leq \frac{5\left(\tau_{2}-\tau_{1}\right)}{72}\left[2\left|\zeta^{\prime}\left(\tau_{2}\right)\right|+\mathcal{F}_{\varkappa, \lambda}^{\aleph}\left(\left|\zeta^{\prime}\left(\tau_{1}\right)\right|-\left|\zeta^{\prime}\left(\tau_{2}\right)\right|\right)\right] .
$$

Remark 9. (i) Choosing $\mathcal{F}_{\varkappa, \lambda}^{\aleph}(\mu-\nu)=(\mu-\nu)$, then inequalities 24 and 26) reduce to inequalities (3.6) and (3.7) in [17].

(ii) Under the assumption of Corollary 3, if we take $\aleph=(1,1, \ldots)$ with $\varkappa=\alpha, \lambda=$ 1, we get the following inequality involving classical Mittag-Leffler function

$$
\begin{aligned}
& \left|\frac{1}{2}\left[\frac{\zeta\left(\tau_{1}\right)+\zeta\left(\tau_{2}\right)}{2}+\zeta\left(\frac{\tau_{1}+\tau_{2}}{2}\right)\right]-\frac{1}{\tau_{2}-\tau_{1}} \int_{\tau_{1}}^{\tau_{2}} \zeta(\mu) d \mu\right| \\
& \leq \frac{\tau_{2}-\tau_{1}}{16}\left[2\left|\zeta^{\prime}\left(\tau_{2}\right)\right|+E_{\alpha}\left(\left|\zeta^{\prime}\left(\tau_{1}\right)\right|-\left|\zeta^{\prime}\left(\tau_{2}\right)\right|\right)\right] \\
& \left|\frac{1}{6}\left[\zeta\left(\tau_{1}\right)+\zeta\left(\tau_{2}\right)+4 \zeta\left(\frac{\tau_{1}+\tau_{2}}{2}\right)\right]-\frac{1}{\tau_{2}-\tau_{1}} \int_{\tau_{1}}^{\tau_{2}} \zeta(\mu) d \mu\right| \\
& \leq \frac{5\left(\tau_{2}-\tau_{1}\right)}{72}\left[2\left|\zeta^{\prime}\left(\tau_{2}\right)\right|+E_{\alpha}\left(\left|\zeta^{\prime}\left(\tau_{1}\right)\right|-\left|\zeta^{\prime}\left(\tau_{2}\right)\right|\right)\right] .
\end{aligned}
$$

Theorem 12. Suppose $\zeta: \mathscr{H} \subseteq \mathscr{R} \rightarrow \mathscr{R}$ be a diff mapp on $\mathscr{H}^{\circ}$ with $\zeta^{\prime} \in L_{1}\left[\tau_{1}, \tau_{2}\right]$, where $\tau_{1}, \tau_{2} \in \mathscr{H}, \tau_{1}<\tau_{2}$. If $\left|\zeta^{\prime}(\mu)\right|^{\ell}$ is Raina-convex function on $\left[\tau_{1}, \tau_{2}\right]$ and $0 \leq \alpha, \beta \leq 1$, then

$$
\begin{aligned}
& \left|\frac{\alpha \zeta\left(\tau_{1}\right)+\beta \zeta\left(\tau_{2}\right)}{2}+\frac{2-\alpha-\beta}{2} \zeta\left(\frac{\tau_{1}+\tau_{2}}{2}\right)-\frac{1}{\tau_{2}-\tau_{1}} \int_{\tau_{1}}^{\tau_{2}} \zeta(\mu) d \mu\right| \\
& \leq \frac{\tau_{2}-\tau_{1}}{4}\left[\frac{1}{2(\ell+1)(\ell+2)}\right]^{\frac{1}{\ell}} \\
& \times\left\{\left[\left(2(\ell+2)(1-\alpha)^{\ell+1}+2(\ell+2) \alpha^{\ell+1}\right)\left|\zeta^{\prime}\left(\tau_{2}\right)\right|^{\ell}\right.\right. \\
& +\left((\ell+3-\alpha)(1-\alpha)^{\ell+1}+(2 \ell+4-\alpha) \alpha^{\ell+1}\right) \\
& \left.\times \mathcal{F}_{\varkappa, \lambda}^{\aleph}\left(\left|\zeta^{\prime}\left(\tau_{1}\right)\right|^{\ell}-\left|\zeta^{\prime}\left(\tau_{2}\right)\right|^{\ell}\right)\right]^{\frac{1}{\ell}}+\left[\left(2(\ell+2)(1-\beta)^{\ell+1}+2(\ell+2) \beta^{\ell+1}\right)\left|\zeta^{\prime}\left(\tau_{2}\right)\right|^{\ell}\right. \\
& \left.\left.+\left(\beta^{\ell+2}+(\ell+1+\beta)(1-\beta)^{\ell+1}\right) \mathcal{F}_{\varkappa, \lambda}^{\aleph}\left(\left|\zeta^{\prime}\left(\tau_{1}\right)\right|^{\ell}-\left|\zeta^{\prime}\left(\tau_{2}\right)\right|^{\ell}\right)\right]^{\frac{1}{\ell}}\right\} .
\end{aligned}
$$

Proof. In case $\ell>1$, using the property of Raina-convexity of $\left|\zeta^{\prime}(\mu)\right|^{\ell}$ on $\left[\tau_{1}, \tau_{2}\right]$ and Hölder's inequality

$$
\left|\frac{\alpha \zeta\left(\tau_{1}\right)+\beta \zeta\left(\tau_{2}\right)}{2}+\frac{2-\alpha-\beta}{2} \zeta\left(\frac{\tau_{1}+\tau_{2}}{2}\right)-\frac{1}{\tau_{2}-\tau_{1}} \int_{\tau_{1}}^{\tau_{2}} \zeta(\mu) d \mu\right|
$$




$$
\begin{aligned}
& \leq \frac{\tau_{2}-\tau_{1}}{4}\left[\int_{0}^{1}(1-\alpha-\kappa)\left|\zeta^{\prime}\left(\kappa \tau_{1}+(1-\kappa) \frac{\tau_{1}+\tau_{2}}{2}\right)\right| d \kappa\right. \\
& \left.+\int_{0}^{1}|\beta-\kappa|\left|\zeta^{\prime}\left(\kappa \frac{\tau_{1}+\tau_{2}}{2}+(1-\kappa) \tau_{2}\right)\right| d \kappa\right] \leq \frac{\tau_{2}-\tau_{1}}{4}\left[( \int _ { 0 } ^ { 1 } d \kappa ) ^ { 1 - \frac { 1 } { \ell } } \left[\int_{0}^{1}|1-\alpha-\kappa|^{\ell} \mid\right.\right. \\
& \left.\times\left(\left|\zeta^{\prime}\left(\tau_{2}\right)\right|^{\ell}+\left(\frac{1+\kappa}{2}\right) \mathcal{F}_{\varkappa, \lambda}^{\aleph}\left(\left|\zeta^{\prime}\left(\tau_{1}\right)\right|^{\ell}-\left|\zeta^{\prime}\left(\tau_{2}\right)\right|^{\ell}\right)\right) d \kappa\right]^{\frac{1}{\ell}} \\
& \left.+\left(\int_{0}^{1} d \kappa\right)^{1-\frac{1}{\ell}}\left[\int_{0}^{1}|\beta-\kappa|^{\ell} \mid\left(\left|\zeta^{\prime}\left(\tau_{2}\right)\right|^{\ell}+\left(\frac{\kappa}{2}\right) \mathcal{F}_{\varkappa, \lambda}^{\aleph}\left(\left|\zeta^{\prime}\left(\tau_{1}\right)\right|^{\ell}-\left|\zeta^{\prime}\left(\tau_{2}\right)\right|^{\ell}\right)\right) d \kappa\right]^{\frac{1}{\ell}}\right] \\
& \leq \frac{\tau_{2}-\tau_{1}}{4}\left[\left[\int_{0}^{1}|1-\alpha-\kappa|^{\ell} \mid\left(\left|\zeta^{\prime}\left(\tau_{2}\right)\right|^{\ell}+\left(\frac{1+\kappa}{2}\right) \mathcal{F}_{\varkappa, \lambda}^{\aleph}\left(\left|\zeta^{\prime}\left(\tau_{1}\right)\right|^{\ell}-\left|\zeta^{\prime}\left(\tau_{2}\right)\right|^{\ell}\right)\right) d \kappa\right]^{\frac{1}{\ell}}\right. \\
& \left.+\left[\int_{0}^{1}|\beta-\kappa|^{\ell} \mid\left(\left|\zeta^{\prime}\left(\tau_{2}\right)\right|^{\ell}+\left(\frac{\kappa}{2}\right) \mathcal{F}_{\varkappa, \lambda}^{\aleph}\left(\left|\zeta^{\prime}\left(\tau_{1}\right)\right|^{\ell}-\left|\zeta^{\prime}\left(\tau_{2}\right)\right|^{\ell}\right)\right) d \kappa\right]^{\frac{1}{\ell}}\right] .
\end{aligned}
$$

By lemma (2) we have

$$
\begin{aligned}
& \int_{0}^{1}|1-\alpha-\kappa|^{\ell}\left(\left|\zeta^{\prime}\left(\tau_{2}\right)\right|^{\ell}+\left(\frac{1+\kappa}{2}\right) \mathcal{F}_{\varkappa, \lambda}^{\aleph}\left(\left|\zeta^{\prime}\left(\tau_{1}\right)\right|^{\ell}-\left|\zeta^{\prime}\left(\tau_{2}\right)\right|^{\ell}\right)\right) d \kappa \\
& =\left(\left|\zeta^{\prime}\left(\tau_{2}\right)\right|^{\ell}+\frac{1}{2} \mathcal{F}_{\varkappa, \lambda}^{\aleph}\left(\left|\zeta^{\prime}\left(\tau_{1}\right)\right|^{\ell}-\left|\zeta^{\prime}\left(\tau_{2}\right)\right|^{\ell}\right)\right) \int_{0}^{1}|1-\alpha-\kappa|^{\ell} d \kappa \\
& +\frac{1}{2} \mathcal{F}_{\varkappa, \lambda}^{\aleph}\left(\left|\zeta^{\prime}\left(\tau_{1}\right)\right|^{\ell}-\left|\zeta^{\prime}\left(\tau_{2}\right)\right|^{\ell}\right) \int_{0}^{1} \kappa|1-\alpha-\kappa|^{\ell} d \kappa \\
& =\left(\left|\zeta^{\prime}\left(\tau_{2}\right)\right|^{\ell}+\frac{1}{2} \mathcal{F}_{\varkappa, \lambda}^{\aleph}\left(\left|\zeta^{\prime}\left(\tau_{1}\right)\right|^{\ell}-\left|\zeta^{\prime}\left(\tau_{2}\right)\right|^{\ell}\right)\right)\left(\frac{(1-\alpha)^{\ell+1}+\alpha^{\ell+1}}{\ell+1}\right) \\
& +\frac{1}{2} \mathcal{F}_{\varkappa, \lambda}^{\aleph}\left(\left|\zeta^{\prime}\left(\tau_{1}\right)\right|^{\ell}-\left|\zeta^{\prime}\left(\tau_{2}\right)\right|^{\ell}\right)\left(\frac{(1-\alpha)^{\ell+2}+(\ell+2-\alpha) \alpha^{\ell+1}}{(\ell+1)(\ell+2)}\right) \\
& =\frac{1}{2(\ell+1)(\ell+2)}\left[2(\ell+2)(1-\alpha)^{\ell+1}+2(\ell+2) \alpha^{\ell+1}\right]\left|\zeta^{\prime}\left(\tau_{2}\right)\right|^{\ell} \\
& +\left[2(\ell+2)(1-\alpha)^{\ell+1}+(\ell+2) \alpha^{\ell+1}+(1-\alpha)^{\ell+2}+(\ell+2-\alpha) \alpha^{\ell+1}\right] \\
& \times \mathcal{F}_{\varkappa, \lambda}^{\aleph}\left(\left|\zeta^{\prime}\left(\tau_{1}\right)\right|^{\ell}-\left|\zeta^{\prime}\left(\tau_{2}\right)\right|^{\ell}\right)=\frac{1}{2(\ell+1)(\ell+2)}\left[2(\ell+2)(1-\alpha)^{\ell+1}\right. \\
& \left.+2(\ell+2) \alpha^{\ell+1}\right]\left|\zeta^{\prime}\left(\tau_{2}\right)\right|^{\ell}+\left[(\ell+3-\alpha)(1-\alpha)^{\ell+1}\right. \\
& \left.\left.+(2 \ell+4-\alpha) \alpha^{\ell+1}\right] \mathcal{F}_{\varkappa, \lambda}^{\aleph}\left(\left|\zeta^{\prime}\left(\tau_{1}\right)\right|^{\ell}-\left|\zeta^{\prime}\left(\tau_{2}\right)\right|^{\ell}\right)\right]
\end{aligned}
$$


and

$$
\begin{aligned}
& \int_{0}^{1}|\beta-\kappa|^{\ell}\left(\left|\zeta^{\prime}\left(\tau_{2}\right)\right|^{\ell}+\left(\frac{\kappa}{2}\right) \mathcal{F}_{\varkappa, \lambda}^{\aleph}\left(\left|\zeta^{\prime}\left(\tau_{1}\right)\right|^{\ell}-\left|\zeta^{\prime}\left(\tau_{2}\right)\right|^{\ell}\right)\right) d \kappa \\
& =\left|\zeta^{\prime}\left(\tau_{2}\right)\right|^{\ell} \int_{0}^{1}|\beta-\kappa|^{\ell} d \kappa+\frac{1}{2} \mathcal{F}_{\varkappa, \lambda}^{\aleph}\left(\left|\zeta^{\prime}\left(\tau_{1}\right)\right|^{\ell}-\left|\zeta^{\prime}\left(\tau_{2}\right)\right|^{\ell}\right) \int_{0}^{1} \kappa|\beta-\kappa|^{\ell} d \kappa \\
& =\left|\zeta^{\prime}\left(\tau_{2}\right)\right|^{\ell}\left(\frac{\beta^{\ell+1}+(1-\beta)^{\ell+1}}{\ell+1}\right)+\frac{1}{2} \mathcal{F}_{\varkappa, \lambda}^{\aleph}\left(\left|\zeta^{\prime}\left(\tau_{1}\right)\right|^{\ell}-\left|\zeta^{\prime}\left(\tau_{2}\right)\right|^{\ell}\right) \\
& \times\left(\frac{\beta^{\ell+2}+(\ell+1+\beta)(1-\beta)^{\ell+1}}{(\ell+1)(\ell+2)}\right) \\
& =\frac{1}{2(\ell+1)(\ell+2)}\left[\left[2(\ell+2)(1-\beta)^{\ell+1}+2(\ell+2) \beta^{\ell+1}\right]\left|\zeta^{\prime}\left(\tau_{2}\right)\right|^{\ell}\right. \\
& \left.+\left[\beta^{\ell+2}+(\ell+1+\beta)(1-\beta)^{\ell+1}\right] \mathcal{F}_{\varkappa, \lambda}^{\aleph}\left(\left|\zeta^{\prime}\left(\tau_{1}\right)\right|^{\ell}-\left|\zeta^{\prime}\left(\tau_{2}\right)\right|^{\ell}\right)\right] .
\end{aligned}
$$

If we put the last two inequalities into inequality 29 , as a result we obtain the inequality 28 for $\ell>1$. If we put $\ell=1$, then the proof is the identitical as that of $(20)$, and the theorem is investigated.

Remark 10. (i) Choosing $\mathcal{F}_{\varkappa, \lambda}^{\aleph}(\mu-\nu)=\mu-\nu$, then the inequality 28) reduces to the inequality (3.8) in [17].

(ii) Under the assumption of Corollary 12, if we take $\aleph=(1,1, \ldots)$ with $\varkappa=\alpha, \lambda=$ 1, we get the following inequality involving classical Mittag-Leffler function

$$
\begin{aligned}
& \left|\frac{\alpha \zeta\left(\tau_{1}\right)+\beta \zeta\left(\tau_{2}\right)}{2}+\frac{2-\alpha-\beta}{2} \zeta\left(\frac{\tau_{1}+\tau_{2}}{2}\right)-\frac{1}{\tau_{2}-\tau_{1}} \int_{\tau_{1}}^{\tau_{2}} \zeta(\mu) d \mu\right| \\
& \leq \frac{\tau_{2}-\tau_{1}}{4}\left[\frac{1}{2(\ell+1)(\ell+2)}\right]^{\frac{1}{\ell}} \\
& \times\left\{\left[\left(2(\ell+2)(1-\alpha)^{\ell+1}+2(\ell+2) \alpha^{\ell+1}\right)\left|\zeta^{\prime}\left(\tau_{2}\right)\right|^{\ell}\right.\right. \\
& +\left((\ell+3-\alpha)(1-\alpha)^{\ell+1}+(2 \ell+4-\alpha) \alpha^{\ell+1}\right) \\
& \left.\times E_{\alpha}\left(\left|\zeta^{\prime}\left(\tau_{1}\right)\right|^{\ell}-\left|\zeta^{\prime}\left(\tau_{2}\right)\right|^{\ell}\right)\right]^{\frac{1}{\ell}}+\left[\left(2(\ell+2)(1-\beta)^{\ell+1}+2(\ell+2) \beta^{\ell+1}\right)\left|\zeta^{\prime}\left(\tau_{2}\right)\right|^{\ell}\right. \\
& \left.\left.+\left(\beta^{\ell+2}+(\ell+1+\beta)(1-\beta)^{\ell+1}\right) E_{\alpha}\left(\left|\zeta^{\prime}\left(\tau_{1}\right)\right|^{\ell}-\left|\zeta^{\prime}\left(\tau_{2}\right)\right|^{\ell}\right)\right]^{\frac{1}{\ell}}\right\}
\end{aligned}
$$

Similarly to Corollaries of Theorem (11), we can obtain the following Corollaries of Theorem 12. 
Corollary 4. Suppose $\zeta: \mathscr{H} \subseteq \mathscr{R} \rightarrow \mathscr{R}$ be a diff mapp on $\mathscr{H}^{o}$ with $\zeta^{\prime} \in L^{1}\left[\tau_{1}, \tau_{2}\right]$ where $\tau_{1}, \tau_{2} \in \mathscr{H}, \tau_{1}<\tau_{2}$. If $\left|\zeta^{\prime}(\mu)\right|^{\ell}$ is Raina-convex function on $\left[\tau_{1}, \tau_{2}\right]$ for $\ell \geq 1$ and $0 \leq \alpha \leq 1$, then

$$
\begin{aligned}
& \left|\frac{\alpha}{2}\left[\zeta\left(\tau_{1}\right)+\zeta\left(\tau_{2}\right)\right]+(1-\alpha) \zeta\left(\frac{\tau_{1}+\tau_{2}}{2}\right)-\frac{1}{\tau_{2}-\tau_{1}} \int_{\tau_{1}}^{\tau_{2}} \zeta(\mu) d \mu\right| \\
& \leq \frac{\tau_{2}-\tau_{1}}{4}\left[\frac{1}{2(\ell+1)(\ell+2)}\right]^{\frac{1}{\ell}}\left\{\left[\left(2(\ell+2)(1-\alpha)^{\ell+1}+2(\ell+2) \alpha^{\ell+1}\right)\left|\zeta^{\prime}\left(\tau_{2}\right)\right|^{\ell}\right.\right. \\
& \left.+\left((\ell+3-\alpha)(1-\alpha)^{\ell+1}+(2 \ell+4-\alpha) \alpha^{\ell+1}\right) \mathcal{F}_{\varkappa, \lambda}^{\aleph}\left(\left|\zeta^{\prime}\left(\tau_{1}\right)\right|^{\ell}-\left|\zeta^{\prime}\left(\tau_{2}\right)\right|^{\ell}\right)\right]^{\frac{1}{\ell}} \\
& +\left[\left(2(\ell+2)(1-\alpha)^{\ell+1}+2(\ell+2) \alpha^{\ell+1}\right)\left|\zeta^{\prime}\left(\tau_{2}\right)\right|^{\ell}\right. \\
& \left.\left.+\left(\alpha^{\ell+2}+(\ell+1+\alpha)(1-\alpha)^{\ell+1}\right) \mathcal{F}_{\varkappa, \lambda}^{\aleph}\left(\left|\zeta^{\prime}\left(\tau_{1}\right)\right|^{\ell}-\left|\zeta^{\prime}\left(\tau_{2}\right)\right|^{\ell}\right)\right]^{\frac{1}{\ell}}\right\}
\end{aligned}
$$

Remark 11. Choosing $\mathcal{F}_{\varkappa, \lambda}^{\aleph}(\mu-\nu)=\mu-\nu$, then inequality (31) collapse to inequality (3.11) in [17].

Corollary 5. Suppose $\zeta: \mathscr{H} \subseteq \mathscr{R} \rightarrow \mathscr{R}$ be a diff mapp on $\mathscr{H}^{\circ}$ with $\zeta^{\prime} \in L^{1}\left[\tau_{1}, \tau_{2}\right]$ where $\tau_{1}, \tau_{2} \in \mathscr{H}, \tau_{1}<\tau_{2}$. If $\left|\zeta^{\prime}(\mu)\right|^{\ell}$ is Raina-convex function on $\left[\tau_{1}, \tau_{2}\right]$ for $\ell \geq 1$ and $0 \leq \alpha, \beta \leq 1$, then

$$
\begin{aligned}
& \left|\frac{1}{2}\left[\frac{\zeta\left(\tau_{1}\right)+\zeta\left(\tau_{2}\right)}{2}+\zeta\left(\frac{\tau_{1}+\tau_{2}}{2}\right)\right]-\frac{1}{\tau_{2}-\tau_{1}} \int_{\tau_{1}}^{\tau_{2}} \zeta(\mu) d \mu\right| \leq \frac{\tau_{2}-\tau_{1}}{8}\left[\frac{1}{4(\ell+1)(\ell+2)}\right]^{\frac{1}{\ell}} \\
& \times\left\{\left[\left((4 \ell+8)\left|\zeta^{\prime}\left(\tau_{2}\right)\right|^{\ell}+(3 \ell+6) \mathcal{F}_{\varkappa, \lambda}^{\aleph}\left(\left|\zeta^{\prime}\left(\tau_{1}\right)\right|^{\ell}-\left|\zeta^{\prime}\left(\tau_{2}\right)\right|^{\ell}\right)\right)\right]^{\frac{1}{\ell}}\right. \\
& \left.+\left[\left((4 \ell+8)\left|\zeta^{\prime}\left(\tau_{2}\right)\right|^{\ell}+(\ell+2) \mathcal{F}_{\varkappa, \lambda}^{\aleph}\left(\left|\zeta^{\prime}\left(\tau_{1}\right)\right|^{\ell}-\left|\zeta^{\prime}\left(\tau_{2}\right)\right|^{\ell}\right)\right)\right]^{\frac{1}{\ell}}\right\} \\
& \left|\frac{1}{6}\left[f\left(\tau_{1}\right)+\zeta\left(\tau_{2}\right)+4 \zeta\left(\frac{\tau_{1}+\tau_{2}}{2}\right)\right]-\frac{1}{\tau_{2}-\tau_{1}} \int_{\tau_{1}}^{\tau_{2}} \zeta(\mu) d \mu\right| \\
& \leq \frac{\tau_{2}-\tau_{1}}{12}\left[\frac{1}{18(\ell+1)(\ell+2)}\right]^{\frac{1}{\ell}}\left\{\left[\left((3 \ell+6) 2^{\ell+2}+6(\ell+2)\right)\left|\zeta^{\prime}\left(\tau_{2}\right)\right|^{\ell}\right.\right. \\
& \left.+\left((3 \ell+8) 2^{\ell+1}+(6 \ell+11) \mathcal{F}_{\varkappa, \lambda}^{\aleph}\left(\left|\zeta^{\prime}\left(\tau_{1}\right)\right|^{\ell}-\left|\zeta^{\prime}\left(\tau_{2}\right)\right|^{\ell}\right)\right)\right]^{\frac{1}{\ell}}+\left[\left((3 \ell+6) 2^{\ell+2}+6(\ell+2)\right)\right. \\
& \left.\left.\left|\zeta^{\prime}\left(\tau_{2}\right)\right|^{\ell}+\left(1+(3 \ell+4) 2^{\ell+1}\right) \mathcal{F}_{\varkappa, \lambda}^{\aleph}\left(\left|\zeta^{\prime}\left(\tau_{1}\right)\right|^{\ell}-\left|\zeta^{\prime}\left(\tau_{2}\right)\right|^{\ell}\right)\right]^{\frac{1}{\ell}}\right\} .
\end{aligned}
$$


Remark 12. (i) Choosing $\mathcal{F}_{\varkappa, \lambda}^{\aleph}(\mu-\nu)=\mu-\nu$, then the inequality (32) reduces to the inequality (3.12) in [17].

(ii) Choosing $\ell=1$ in Corollary (4), then we get Corollary (3).

(iii) Under the assumption of Corollary 5 , if we take $\aleph=(1,1, \ldots)$ with $\varkappa=\alpha, \lambda=$ 1, we get the following inequality involving classical Mittag-Leffler function

$$
\begin{aligned}
& \left|\frac{1}{2}\left[\frac{\zeta\left(\tau_{1}\right)+\zeta\left(\tau_{2}\right)}{2}+\zeta\left(\frac{\tau_{1}+\tau_{2}}{2}\right)\right]-\frac{1}{\tau_{2}-\tau_{1}} \int_{\tau_{1}}^{\tau_{2}} f(\mu) d \mu\right| \leq \frac{\tau_{2}-\tau_{1}}{8}\left[\frac{1}{4(\ell+1)(\ell+2)}\right]^{\frac{1}{\ell}} \\
& \times\left\{\left[\left((4 \ell+8)\left|\zeta^{\prime}\left(\tau_{2}\right)\right|^{\ell}+(3 \ell+6) E_{\alpha}\left(\left|\zeta^{\prime}\left(\tau_{1}\right)\right|^{\ell}-\left|\zeta^{\prime}\left(\tau_{2}\right)\right|^{\ell}\right)\right)\right]^{\frac{1}{\ell}}\right. \\
& \left.+\left[\left((4 \ell+8)\left|\zeta^{\prime}\left(\tau_{2}\right)\right|^{\ell}+(\ell+2) E_{\alpha}\left(\left|\zeta^{\prime}\left(\tau_{1}\right)\right|^{\ell}-\left|\zeta^{\prime}\left(\tau_{2}\right)\right|^{\ell}\right)\right)\right]^{\frac{1}{\ell}}\right\} \\
& \left|\frac{1}{6}\left[\zeta\left(\tau_{1}\right)+\zeta\left(\tau_{2}\right)+4 \zeta\left(\frac{\tau_{1}+\tau_{2}}{2}\right)\right]-\frac{1}{\tau_{2}-\tau_{1}} \int_{\tau_{1}}^{\tau_{2}} \zeta(\mu) d \mu\right| \\
& \leq \frac{1}{12}\left[\frac{1}{18(\ell+1)(\ell+2)}\right]^{\frac{1}{\ell}}\left\{\left[\left((3 \ell+6) 2^{\ell+2}+6(\ell+2)\right)\left|\zeta^{\prime}\left(\tau_{2}\right)\right|^{\ell}\right.\right. \\
& \left.+\left((3 \ell+8) 2^{\ell+1}+(6 \ell+11) E_{\alpha}\left(\left|\zeta^{\prime}\left(\tau_{1}\right)\right|^{\ell}-\left|\zeta^{\prime}\left(\tau_{2}\right)\right|^{\ell}\right)\right)\right]^{\frac{1}{\ell}}+\left[\left((3 \ell+6) 2^{\ell+2}+6(\ell+2)\right)\right. \\
& \left.\left.\left|\zeta^{\prime}\left(\tau_{2}\right)\right|^{\ell}+\left(1+(3 \ell+4) 2^{\ell+1}\right) E_{\alpha}\left(\left|\zeta^{\prime}\left(\tau_{1}\right)\right|^{\ell}-\left|\zeta^{\prime}\left(\tau_{2}\right)\right|^{\ell}\right)\right]^{\frac{1}{\ell}}\right\} .
\end{aligned}
$$

For further results, we highlight the below Lemma which is proved in 11.

Lemma 3. 11] Suppose $\zeta: \mathscr{H} \subseteq \mathscr{R} \rightarrow \mathscr{R}$ be a diff mapp on $\mathscr{H}^{\circ}$ with $\zeta^{\prime} \in$ $L^{1}\left[\tau_{1}, \tau_{2}\right]$ where $\tau_{1}, \tau_{2} \in \mathscr{H}$ and $\tau_{1}<\tau_{2}$, then

$$
\begin{aligned}
& \frac{1}{\tau_{2}-\tau_{1}} \int_{\tau_{1}}^{\tau_{2}} \zeta(\mu) d \mu-\zeta\left(\frac{\tau_{1}+\tau_{2}}{2}\right)=\frac{\left(\tau_{2}-\tau_{1}\right)^{2}}{16} \\
& \times\left[\int_{0}^{1} \kappa^{2} \zeta^{\prime \prime}\left(\kappa \frac{\tau_{1}+\tau_{2}}{2}+(1-\kappa) \tau_{1}\right) d \kappa+\int_{0}^{1}(\kappa-1)^{2} \zeta^{\prime \prime}\left(\kappa \tau_{2}+(1-\kappa) \frac{\tau_{1}+\tau_{2}}{2}\right) d \kappa\right] .
\end{aligned}
$$

Theorem 13. Suppose $\zeta: \mathscr{H} \subseteq \mathscr{R} \rightarrow \mathscr{R}$ be a diff mapp on $\mathscr{H}^{\circ}$ with $\zeta^{\prime \prime} \in$ $L^{1}\left[\tau_{1}, \tau_{2}\right]$, where $\tau_{1}, \tau_{2} \in \mathscr{H}$ and $\tau_{1}<\tau_{2}$. If $\left|\zeta^{\prime \prime}(\mu)\right|$ is Raina-convex function on $\left[\tau_{1}, \tau_{2}\right]$, then

$$
\left|\zeta\left(\frac{\tau_{1}+\tau_{2}}{2}\right)-\frac{1}{\tau_{2}-\tau_{1}} \int_{\tau_{1}}^{\tau_{2}} \zeta(\mu) d \mu\right| \leq \frac{\left(\tau_{2}-\tau_{1}\right)^{2}}{16}\left[\frac{1}{3}\left(\left|\zeta^{\prime \prime}\left(\tau_{1}\right)\right|+\left|\zeta^{\prime \prime}\left(\frac{\tau_{1}+\tau_{2}}{2}\right)\right|\right)\right.
$$




$$
\left.+\frac{1}{4}\left(\mathcal{F}_{\varkappa, \lambda}^{\aleph}\left(\left|\zeta^{\prime \prime}\left(\frac{\tau_{1}+\tau_{2}}{2}\right)\right|-\left|\zeta^{\prime \prime}\left(\tau_{1}\right)\right|\right)\right)+\frac{1}{3} \mathcal{F}_{\varkappa, \lambda}^{\aleph}\left(\left|\zeta^{\prime \prime}\left(\tau_{2}\right)\right|-\left|\zeta^{\prime \prime}\left(\frac{\tau_{1}+\tau_{2}}{2}\right)\right|\right)\right] .
$$

Proof. From lemma (3), we have

$$
\begin{aligned}
& \left|\zeta\left(\frac{\tau_{1}+\tau_{2}}{2}\right)-\frac{1}{\tau_{2}-\tau_{1}} \int_{\tau_{1}}^{\tau_{2}} \zeta(\mu) d \mu\right| \leq \frac{\left(\tau_{2}-\tau_{1}\right)^{2}}{16}\left[\int_{0}^{1} \kappa^{2}\left|\zeta^{\prime \prime}\left(\kappa \frac{\tau_{1}+\tau_{2}}{2}+(1-\kappa) \tau_{1}\right)\right| d \kappa\right. \\
& \left.+\int_{0}^{1}(\kappa-1)^{2}\left|\zeta^{\prime \prime}\left(\kappa \tau_{2}+(1-\kappa) \frac{\tau_{1}+\tau_{2}}{2}\right)\right| d \kappa\right] \\
& \leq \frac{\left(\tau_{2}-\tau_{1}\right)^{2}}{16}\left[\int_{0}^{1} \kappa^{2}\left(\left|\zeta^{\prime \prime}\left(\tau_{1}\right)\right|+\kappa \mathcal{F}_{\rho, \lambda}^{\sigma}\left(\left|\zeta^{\prime \prime}\left(\frac{\tau_{1}+\tau_{2}}{2}\right)\right|-\left|\zeta^{\prime \prime}\left(\tau_{1}\right)\right|\right)\right) d \kappa\right] \\
& +\frac{\left(\tau_{2}-\tau_{1}\right)^{2}}{16}\left[\int_{0}^{1}(\kappa-1)^{2}\left(\left|\zeta^{\prime \prime}\left(\frac{\tau_{1}+\tau_{2}}{2}\right)\right|+\kappa \mathcal{F}_{\varkappa, \lambda}^{\aleph}\left(\left|\zeta^{\prime \prime}\left(\tau_{2}\right)\right|-\left|\zeta^{\prime \prime}\left(\frac{\tau_{1}+\tau_{2}}{2}\right)\right|\right)\right) d \kappa\right] \\
& =\frac{\left(\tau_{2}-\tau_{1}\right)^{2}}{16}\left[\frac{1}{3}\left|\zeta^{\prime \prime}\left(\tau_{1}\right)\right|+\frac{1}{3}\left|\zeta^{\prime \prime}\left(\frac{\tau_{1}+\tau_{2}}{2}\right)\right|+\frac{1}{4} \mathcal{F}_{\varkappa, \lambda}^{\aleph}\left(\left|\zeta^{\prime \prime}\left(\frac{\tau_{1}+\tau_{2}}{2}\right)\right|-\left|\zeta^{\prime \prime}\left(\tau_{1}\right)\right|\right)\right. \\
& \left.+\frac{1}{12} \mathcal{F}_{\varkappa, \lambda}^{\aleph}\left(\left|\zeta^{\prime \prime}\left(\tau_{2}\right)\right|-\left|\zeta^{\prime \prime}\left(\frac{\tau_{1}+\tau_{2}}{2}\right)\right|\right)\right]=\frac{\left(\tau_{2}-\tau_{1}\right)^{2}}{16}\left[\frac{1}{3}\left(\left|\zeta^{\prime \prime}\left(\tau_{1}\right)\right|+\left|\zeta^{\prime \prime}\left(\frac{\tau_{1}+\tau_{2}}{2}\right)\right|\right)\right. \\
& \left.+\frac{1}{4} \mathcal{F}_{\varkappa, \lambda}^{\aleph}\left(\left|\zeta^{\prime \prime}\left(\frac{\tau_{1}+\tau_{2}}{2}\right)\right|-\left|\zeta^{\prime \prime}\left(\tau_{1}\right)\right|\right)+\frac{1}{3} \mathcal{F}_{\varkappa, \lambda}^{\aleph}\left(\left|\zeta^{\prime \prime}\left(\tau_{2}\right)\right|-\left|\zeta^{\prime \prime}\left(\frac{\tau_{1}+\tau_{2}}{2}\right)\right|\right)\right] .
\end{aligned}
$$

Remark 13. (i) Choosing $\mathcal{F}_{\varkappa, \lambda}^{\aleph}(\mu-\nu)=\mu-\nu$, then inequality (35) reduce to inequality (5).

(ii) Under the assumption of Theorem 13 , if we take $\aleph=(1,1, \ldots)$ with $\varkappa=\alpha, \lambda=$ 1, we get the following inequality involving classical Mittag-Leffler function

$$
\begin{aligned}
& \left|\zeta\left(\frac{\tau_{1}+\tau_{2}}{2}\right)-\frac{1}{\tau_{2}-\tau_{1}} \int_{\tau_{1}}^{\tau_{2}} \zeta(\mu) d \mu\right| \leq \frac{\left(\tau_{2}-\tau_{1}\right)^{2}}{16}\left[\frac{1}{3}\left(\left|\zeta^{\prime \prime}\left(\tau_{1}\right)\right|+\left|\zeta^{\prime \prime}\left(\frac{\tau_{1}+\tau_{2}}{2}\right)\right|\right)\right. \\
& \left.+\frac{1}{4}\left(E_{\alpha}\left(\left|\zeta^{\prime \prime}\left(\frac{\tau_{1}+\tau_{2}}{2}\right)\right|-\left|\zeta^{\prime \prime}\left(\tau_{1}\right)\right|\right)\right)+\frac{1}{3} E_{\alpha}\left(\left|\zeta^{\prime \prime}\left(\tau_{2}\right)\right|-\left|\zeta^{\prime \prime}\left(\frac{\tau_{1}+\tau_{2}}{2}\right)\right|\right)\right] .
\end{aligned}
$$

Theorem 14. Suppose $\zeta: \mathscr{H} \subseteq \mathscr{R} \rightarrow \mathscr{R}$ be a diff mapp on $\mathscr{H}^{\circ}$ with $\zeta^{\prime \prime} \in$ $L^{1}\left[\tau_{1}, \tau_{2}\right]$, where $\tau_{1}, \tau_{2} \in \mathscr{H}$ and $\tau_{1}<\tau_{2}$. If $\left|\zeta^{\prime \prime}(\mu)\right|^{\ell}$ for $\ell \geq 1$ with $\frac{1}{p}+\frac{1}{\ell}=1$ is Raina-convex function on $\left[\tau_{1}, \tau_{2}\right]$, then

$$
\left.\mid \zeta\left(\frac{\tau_{1}+\tau_{2}}{2}\right)-\frac{1}{\tau_{2}-\tau_{1}} \int_{\tau_{1}}^{\tau_{2}} \zeta(\mu) d \mu\right) \mid \leq \frac{\left(\tau_{2}-\tau_{1}\right)^{2}}{16}\left(\frac{1}{3}\right)^{\frac{1}{p}}
$$




$$
\begin{aligned}
& \times\left[\left(\frac{1}{3}\left|\zeta^{\prime \prime}\left(\tau_{1}\right)\right|^{\ell}+\frac{1}{4} \mathcal{F}_{\varkappa, \lambda}^{\aleph}\left(\left|\zeta^{\prime \prime}\left(\frac{\tau_{1}+\tau_{2}}{2}\right)\right|^{\ell}-\left|\zeta^{\prime \prime}\left(\tau_{1}\right)\right|^{\ell}\right)\right)^{\frac{1}{\ell}}\right. \\
& \left.+\left(\frac{1}{3}\left|\zeta^{\prime \prime}\left(\frac{\tau_{1}+\tau_{2}}{2}\right)\right|^{\ell}+\frac{1}{12} \mathcal{F}_{\varkappa, \lambda}^{\aleph}\left(\left|\zeta^{\prime \prime}\left(\tau_{2}\right)\right|^{\ell}-\left|\zeta^{\prime \prime}\left(\frac{\tau_{1}+\tau_{2}}{2}\right)\right|^{\ell}\right)\right)^{\frac{1}{\ell}}\right] .
\end{aligned}
$$

Proof. If $p \geq 1$, using lemma (3) and power Mean Inequality, then

$$
\begin{aligned}
& \left.\mid \zeta\left(\frac{\tau_{1}+\tau_{2}}{2}\right)-\frac{1}{\tau_{2}-\tau_{1}} \int_{\tau_{1}}^{\tau_{2}} \zeta(\mu) d \mu\right) \mid \leq \frac{\left(\tau_{2}-\tau_{1}\right)^{2}}{16}\left[\int_{0}^{1} \kappa^{2}\left|\zeta^{\prime \prime}\left(\kappa \frac{\tau_{1}+\tau_{2}}{2}+(1-\kappa) \tau_{1}\right)\right| d \kappa\right. \\
& \left.+\int_{0}^{1}(\kappa-1)^{2}\left|\zeta^{\prime \prime}\left(\kappa \tau_{2}+(1-\kappa) \frac{\tau_{1}+\tau_{2}}{2}\right)\right| d \kappa\right] \\
& \leq \frac{\left(\tau_{2}-\tau_{1}\right)^{2}}{16}\left(\int_{0}^{1} \kappa^{2} d \kappa\right)^{\frac{1}{p}}\left[\int_{0}^{1} \kappa^{2}\left|\zeta^{\prime \prime}\left(\kappa \frac{\tau_{1}+\tau_{2}}{2}+(1-\kappa) \tau_{1}\right)\right|^{\ell} d \kappa\right]^{\frac{1}{\ell}} \\
& +\frac{\left(\tau_{2}-\tau_{1}\right)^{2}}{16}\left(\int_{0}^{1}(\kappa-1)^{2} d \kappa\right)^{\frac{1}{p}}\left(\int_{0}^{1}(\kappa-1)^{2}\left|\zeta^{\prime \prime}\left(\kappa \tau_{2}+(1-\kappa) \frac{\tau_{1}+\tau_{2}}{2}\right)\right|^{\ell} d \kappa\right)^{\frac{1}{\ell}}
\end{aligned}
$$

Because $\left|\zeta^{\prime \prime}\right|^{\ell}$ is Raina-convex function, we have

$$
\begin{aligned}
\int_{0}^{1} \kappa^{2}\left|\zeta^{\prime \prime}\left(\kappa \frac{\tau_{1}+\tau_{2}}{2}+(1-\kappa) \tau_{1}\right)\right|^{\ell} d \kappa & \leq \frac{1}{3}\left|\zeta^{\prime \prime}\left(\tau_{1}\right)\right|^{\ell} \\
& +\frac{1}{4}\left(\mathcal{F}_{\varkappa, \lambda}^{\aleph}\left(\left|\zeta^{\prime \prime}\left(\frac{\tau_{1}+\tau_{2}}{2}\right)\right|^{\ell}-\left|\zeta^{\prime \prime}\left(\tau_{2}\right)\right|^{\ell}\right)\right)
\end{aligned}
$$

and

$$
\begin{aligned}
\int_{0}^{1}(\kappa-1)^{2}\left|\zeta^{\prime \prime}\left(\kappa \tau_{2}+(1-\kappa) \frac{\tau_{1}+\tau_{2}}{2}\right)\right|^{\ell} d \kappa & \leq \frac{1}{3}\left|\zeta^{\prime \prime}\left(\frac{\tau_{1}+\tau_{2}}{2}\right)\right|^{\ell} \\
& +\frac{1}{12} \mathcal{F}_{\varkappa, \lambda}^{\aleph}\left(\left|\zeta^{\prime \prime}\left(\tau_{2}\right)\right|^{\ell}-\left|\zeta^{\prime \prime}\left(\frac{\tau_{1}+\tau_{2}}{2}\right)\right|^{\ell}\right)
\end{aligned}
$$

Therefore we have

$$
\begin{aligned}
& \left|\zeta\left(\frac{\tau_{1}+\tau_{2}}{2}\right)-\frac{1}{\tau_{2}-\tau_{1}} \int_{\tau_{1}}^{\tau_{2}} \zeta(\mu) d \mu\right| \leq \frac{\left(\tau_{2}-\tau_{1}\right)^{2}}{16}\left(\frac{1}{3}\right)^{\frac{1}{p}} \\
& \times\left[\left(\frac{1}{3}\left|\zeta^{\prime \prime}\left(\tau_{1}\right)\right|^{\ell}+\frac{1}{4} \mathcal{F}_{\varkappa, \lambda}^{\aleph}\left(\left|\zeta^{\prime \prime}\left(\frac{\tau_{1}+\tau_{2}}{2}\right)\right|-\left|\zeta^{\prime \prime}\left(\tau_{1}\right)\right|^{\ell}\right)\right)^{\frac{1}{\ell}}\right. \\
& \left.+\left(\frac{1}{3}\left|\zeta^{\prime \prime}\left(\frac{\tau_{1}+\tau_{2}}{2}\right)\right|^{\ell}+\frac{1}{12} \mathcal{F}_{\varkappa, \lambda}^{\aleph}\left(\left|\zeta^{\prime \prime}\left(\tau_{2}\right)\right|^{\ell}-\left|\zeta^{\prime \prime}\left(\frac{\tau_{1}+\tau_{2}}{2}\right)\right|^{\ell}\right)\right)^{\frac{1}{\ell}}\right] .
\end{aligned}
$$

Remark 14. (i) Choosing $\mathcal{F}_{\varkappa, \lambda}^{\aleph}(\mu-\nu)=\mu-\nu$, then inequality (37) reduce to inequality (6). 
(ii) Under the assumption of Theorem 14, if we take $\aleph=(1,1, \ldots)$ with $\varkappa=\alpha, \lambda=$ 1, we get the following inequality involving classical Mittag-Leffler function

$$
\begin{aligned}
& \left.\mid \zeta\left(\frac{\tau_{1}+\tau_{2}}{2}\right)-\frac{1}{\tau_{2}-\tau_{1}} \int_{\tau_{1}}^{\tau_{2}} \zeta(\mu) d \mu\right) \mid \leq \frac{\left(\tau_{2}-\tau_{1}\right)^{2}}{16}\left(\frac{1}{3}\right)^{\frac{1}{p}} \\
& \times\left[\left(\frac{1}{3}\left|\zeta^{\prime \prime}\left(\tau_{1}\right)\right|^{\ell}+\frac{1}{4} E_{\alpha}\left(\left|\zeta^{\prime \prime}\left(\frac{\tau_{1}+\tau_{2}}{2}\right)\right|^{\ell}-\left|\zeta^{\prime \prime}\left(\tau_{1}\right)\right|^{\ell}\right)\right)^{\frac{1}{\ell}}\right. \\
& \left.+\left(\frac{1}{3}\left|\zeta^{\prime \prime}\left(\frac{\tau_{1}+\tau_{2}}{2}\right)\right|^{\ell}+\frac{1}{12} E_{\alpha}\left(\left|\zeta^{\prime \prime}\left(\tau_{2}\right)\right|^{\ell}-\left|\zeta^{\prime \prime}\left(\frac{\tau_{1}+\tau_{2}}{2}\right)\right|^{\ell}\right)\right)^{\frac{1}{\ell}}\right] .
\end{aligned}
$$

\section{Applications}

In this section, we recall the following special means for two positive real numbers $\tau_{1}, \tau_{2}$ where $\tau_{1}<\tau_{2}$ :

(1) The arithmetic mean

$$
A=A\left(\tau_{1}, \tau_{2}\right)=\frac{\tau_{1}+\tau_{2}}{2} .
$$

(2) The geometric mean

$$
G=G\left(\tau_{1}, \tau_{2}\right)=\sqrt{\tau_{1} \tau_{2}}
$$

(3) The harmonic mean

$$
H=H\left(\tau_{1}, \tau_{2}\right)=\frac{2 \tau_{1} \tau_{2}}{\tau_{1}+\tau_{2}} .
$$

(4) The p-logarithmic mean

$$
L_{p}=L_{p}\left(\tau_{1}, \tau_{2}\right)=\left(\frac{\tau_{2}^{p+1}-\tau_{1}^{p+1}}{(p+1)\left(\tau_{2}-\tau_{1}\right)}\right)^{\frac{1}{p}}, \quad p \in \mathbb{R} \backslash\{0\} .
$$

(5) The identric mean

$$
I=I\left(\tau_{1}, \tau_{2}\right)=\frac{1}{e}\left(\frac{\tau_{2}^{\tau_{2}}}{\tau_{1}^{\tau_{1}}}\right)^{\frac{1}{\tau_{2}-\tau_{1}}}
$$

(6) The heronian mean

$$
H_{w, s}\left(\tau_{1}, \tau_{2}\right)= \begin{cases}{\left[\frac{\tau_{2}^{s}+w\left(\tau_{1} \tau_{2}\right)^{\frac{s}{2}}+\tau_{2}^{s}}{w+2}\right]^{\frac{1}{s}},} & \text { if } s \neq 0 \\ \sqrt{\tau_{1} \tau_{2}}, & \text { if } s=0 .\end{cases}
$$

These means have a lot of applications in areas and different type of numerical approximations. However, the following simple relationship are known in the literature.

If we choose $\zeta(\mu)=\mu^{s}$ for $s \neq 0$ and $x>0$ in Theorems (11) and 12), as a result we get the following inequalities for means. 
Proposition 1. Let $\tau_{1}>0, \tau_{2}>0, \tau_{1} \neq \tau_{2}, \ell \geq 1$ and either $s>1$ and $(s-1) \ell \geq 1$ or $s<0$ Then

$$
\begin{aligned}
& \left|A\left(\alpha \tau_{1}^{s}, \beta \tau_{2}^{s}\right)+\frac{2-\alpha-\beta}{2} A^{s}\left(\tau_{1}, \tau_{2}\right)-L^{s}\left(\tau_{1}, \tau_{2}\right)\right| \leq \frac{\tau_{2}-\tau_{1}}{8}\left(\frac{1}{6}\right)^{\frac{1}{\ell}} \\
& \times\left[( 1 - 2 \alpha + 2 \alpha ^ { 2 } ) ^ { 1 - \frac { 1 } { \ell } } \left[\left(6-12 \alpha+12 \alpha^{2}\right)\left|s \tau_{2}^{s-1}\right|^{\ell}+\left(4-9 \alpha+12 \alpha^{2}-2 \alpha^{3}\right)\right.\right. \\
& \left.\times \mathcal{F}_{\varkappa, \lambda}^{\aleph}\left(\left|s \tau_{1}^{s-1}\right|^{\ell}-\left|s \tau_{2}^{s-1}\right|^{\ell}\right)\right]^{\frac{1}{\ell}}+\left(1-2 \beta+2 \beta^{2}\right)^{1-\frac{1}{\ell}}\left[\left(6-12 \beta+12 \beta^{2}\right)\left|s \tau_{2}^{s-1}\right|^{\ell}\right. \\
& \left.\left.+\left(2-3 \beta+2 \beta^{3}\right) \mathcal{F}_{\varkappa, \lambda}^{\aleph}\left(\left|s \tau_{1}^{s-1}\right|^{\ell}-\left|s \tau_{2}^{s-1}\right|^{\ell}\right)\right]^{\frac{1}{\ell}}\right]
\end{aligned}
$$

Remark 15. Under the assumption of Proposition 1, if we take $\aleph=(1,1, \ldots)$ with $\varkappa=\alpha, \lambda=1$, we get the following inequality involving classical Mittag-Leffler function

$$
\begin{aligned}
& \left|A\left(\alpha \tau_{1}^{s}, \beta \tau_{2}^{s}\right)+\frac{2-\alpha-\beta}{2} A^{s}\left(\tau_{1}, \tau_{2}\right)-L^{s}\left(\tau_{1}, \tau_{2}\right)\right| \leq \frac{\tau_{2}-\tau_{1}}{8}\left(\frac{1}{6}\right)^{\frac{1}{\ell}} \\
& \times\left[( 1 - 2 \alpha + 2 \alpha ^ { 2 } ) ^ { 1 - \frac { 1 } { \ell } } \left[\left(6-12 \alpha+12 \alpha^{2}\right)\left|s \tau_{2}^{s-1}\right|^{\ell}+\left(4-9 \alpha+12 \alpha^{2}-2 \alpha^{3}\right)\right.\right. \\
& \left.\times E_{\alpha}\left(\left|s \tau_{1}^{s-1}\right|^{\ell}-\left|s \tau_{2}^{s-1}\right|^{\ell}\right)\right]^{\frac{1}{\ell}}+\left(1-2 \beta+2 \beta^{2}\right)^{1-\frac{1}{\ell}}\left[\left(6-12 \beta+12 \beta^{2}\right)\left|s \tau_{2}^{s-1}\right|^{\ell}\right. \\
& \left.\left.+\left(2-3 \beta+2 \beta^{3}\right) \times E_{\alpha}\left(\left|s \tau_{1}^{s-1}\right|^{\ell}-\left|s \tau_{2}^{s-1}\right|^{\ell}\right)\right]^{\frac{1}{\ell}}\right]
\end{aligned}
$$

Proposition 2. Let $\tau_{1}>0, \tau_{2}>0, \tau_{1} \neq \tau_{2}, \ell \geq 1$ and either $s>1$ and $(s-1) \ell \geq 1$ or $s<0$

$$
\begin{aligned}
& \left|A\left(\alpha \tau_{1}^{s}, \beta \tau_{2}^{s}\right)+\frac{2-\alpha-\beta}{2} A^{s}\left(\tau_{1}, \tau_{2}\right)-L^{s}\left(\tau_{1}, \tau_{2}\right)\right| \leq \frac{\left(\tau_{2}-\tau_{1}\right)}{4}\left[\frac{1}{2(\ell+1)(\ell+2)}\right]^{\frac{1}{\ell}} \\
& \times\left[\left[\left(\left[2(\ell+2)(1-\alpha)^{\ell+1}+2(\ell+2) \alpha^{\ell+1}\right]\right)\left|s \tau_{2}^{s-1}\right|^{\ell}+\left[(\ell+3-\alpha)(1-\alpha)^{\ell+1}\right.\right.\right. \\
& \left.\left.+(2 \ell+4-\alpha) \alpha^{\ell+1}\right] \mathcal{F}_{\varkappa, \lambda}^{\aleph}\left(\left|s \tau_{1}^{s-1}\right|^{\ell}-\left|s \tau_{2}^{s-1}\right|^{\ell}\right)\right]^{\frac{1}{\ell}}+\left[\left(2(\ell+2)(1-\beta)^{\ell+1}\right.\right. \\
& \left.\left.\left.+2(\ell+2) \beta^{q+1}\right)\left|s \tau_{2}^{s-1}\right|^{\ell}+\left(\beta^{\ell+2}+(\ell+1+\beta)(1-\beta)^{\ell+1}\right) \mathcal{F}_{\varkappa, \lambda}^{\aleph}\left(\left|s \tau_{1}^{s-1}\right|^{\ell}-\left|s \tau_{2}^{s-1}\right|^{\ell}\right)\right]^{\frac{1}{\ell}}\right] .
\end{aligned}
$$


If we choose $\zeta(\mu)=\ln \mu$ for $\mu>0$ in theorems (11) and 12 , as a result we get the following inequalities for mean.

Proposition 3. For $\tau_{1}>0, \tau_{2}>0, \tau_{1} \neq \tau_{2}$ and $\ell \geq 1$, we have

$$
\begin{aligned}
& \left|\frac{\ln G^{2}\left(\tau_{1}^{\alpha}, \tau_{2}^{\beta}\right)}{2}+\frac{2-\alpha-\beta}{2} \ln A\left(\tau_{1}, \tau_{2}\right)-\ln I\left(\tau_{1}, \tau_{2}\right)\right| \leq \frac{\tau_{2}-\tau_{1}}{8}\left(\frac{1}{6}\right)^{\frac{1}{\ell}}\left[\left(1-2 \alpha+2 \alpha^{2}\right)^{1-\frac{1}{\ell}}\right. \\
& \times\left[\left(6-12 \alpha+12 \alpha^{2}\right)\left(\frac{1}{\tau_{2}}\right)^{\ell}+\left(4-9 \alpha+12 \alpha^{2}-2 \alpha^{3}\right) \mathcal{F}_{\varkappa, \lambda}^{\aleph}\left(\left(\frac{1}{\tau_{1}}\right)^{\ell}-\left(\frac{1}{\tau_{2}}\right)^{\ell}\right)\right]^{\frac{1}{\ell}}(42) \\
& \left.+\left(1-2 \beta+2 \beta^{2}\right)^{1-\frac{1}{\ell}}\left[\left(6-12 \beta+12 \beta^{2}\right)\left(\frac{1}{\tau_{2}}\right)^{\ell}+\left(2-3 \beta+2 \beta^{3}\right) \mathcal{F}_{\varkappa, \lambda}^{\aleph}\left(\left(\frac{1}{\tau_{1}}\right)^{\ell}-\left(\frac{1}{\tau_{2}}\right)^{\ell}\right)\right]^{\frac{1}{\ell}}\right] .
\end{aligned}
$$

Remark 16. Under the assumption of Proposition 3. if we take $\aleph=(1,1, \ldots)$ with $\varkappa=\alpha, \lambda=1$, we get the following inequality involving classical Mittag-Leffler function

$$
\begin{aligned}
& \left|\frac{\ln G^{2}\left(\tau_{1}^{\alpha}, \tau_{2}^{\beta}\right)}{2}+\frac{2-\alpha-\beta}{2} \ln A\left(\tau_{1}, \tau_{2}\right)-\ln I\left(\tau_{1}, \tau_{2}\right)\right| \leq \frac{\tau_{2}-\tau_{1}}{8}\left(\frac{1}{6}\right)^{\frac{1}{\ell}} \\
& \times\left[\left(1-2 \alpha+2 \alpha^{2}\right)^{1-\frac{1}{\ell}}\left[\left(6-12 \alpha+12 \alpha^{2}\right)\left(\frac{1}{\tau_{2}}\right)^{\ell}+\left(4-9 \alpha+12 \alpha^{2}-2 \alpha^{3}\right) E_{\alpha}\left(\left(\frac{1}{\tau_{1}}\right)^{\ell}-\left(\frac{1}{\tau_{2}}\right)^{\ell}\right)\right]^{\frac{1}{\ell}}\right. \\
& \left.+\left(1-2 \beta+2 \beta^{2}\right)^{1-\frac{1}{\ell}}\left[\left(6-12 \beta+12 \beta^{2}\right)\left(\frac{1}{\tau_{2}}\right)^{\ell}+\left(2-3 \beta+2 \beta^{3}\right) E_{\alpha}\left(\left(\frac{1}{\tau_{1}}\right)^{\ell}-\left(\frac{1}{\tau_{2}}\right)^{\ell}\right)\right]^{\frac{1}{\ell}}\right] .
\end{aligned}
$$

Proposition 4. For $\tau_{1}>0, \tau_{2}>0, \tau_{1} \neq \tau_{2}$ and $\ell \geq 1$, we have

$$
\begin{aligned}
& \left|\frac{\ln G^{2}\left(\tau_{1}^{\alpha}, \tau_{2}^{\beta}\right)}{2}+\frac{2-\alpha-\beta}{2} \ln A\left(\tau_{1}, \tau_{2}\right)-\ln I\left(\tau_{1}, \tau_{2}\right)\right| \leq \frac{\tau_{2}-\tau_{1}}{4}\left[\frac{1}{2(\ell+1)(\ell+2)}\right]^{\frac{1}{\ell}} \\
& \times\left[\left[\left(2(\ell+2)(1-\alpha)^{\ell+1}+2(\ell+2) \alpha^{\ell+1}\right)\left(\frac{1}{\tau_{2}}\right)^{\ell}+\left[(q+3-\alpha)(1-\alpha)^{\ell+1}+(2 \ell+4-\alpha) \alpha^{\ell+1}\right]\right.\right. \\
& \left.\times \mathcal{F}_{\varkappa, \lambda}^{\aleph}\left(\left(\frac{1}{\tau_{1}}\right)^{\ell}-\left(\frac{1}{\tau_{2}}\right)^{\ell}\right)\right]^{\frac{1}{\ell}}+\left[\left(2(\ell+2)(1-\beta)^{\ell+1}+2(\ell+2) \beta^{\ell+1}\right)\left(\frac{1}{\tau_{2}}\right)^{\ell}\right. \\
& \left.\left.+\left((q+1+\beta)(1-\beta)^{q+1}+\beta^{q+2}\right) \mathcal{F}_{\varkappa, \lambda}^{\aleph}\left(\left(\frac{1}{\tau_{1}}\right)^{\ell}-\left(\frac{1}{\tau_{2}}\right)^{\ell}\right)\right]^{\frac{1}{\ell}}\right] .
\end{aligned}
$$

Finally,

Proposition 5. For $\tau_{2}>\tau_{1}>0, \tau_{1} \neq \tau_{2}, w \geq 0$ and $s \geq 4$ or $0 \neq s<1$, we have

$$
\left|\frac{H_{w, s}^{s}\left(\tau_{1}, \tau_{2}\right)}{H\left(\tau_{1}^{s}, \tau_{2}^{s}\right)}+H_{w,\left(\frac{s}{2}+1\right)}^{\frac{s}{2}+1}\left(\frac{\tau_{2}}{\tau_{1}}+\frac{\tau_{1}}{\tau_{2}}, 1\right)-H_{w, s}^{s}\left(\frac{L\left(\tau_{1}^{2}, \tau_{2}^{2}\right)}{G^{2}\left(\tau_{1}, \tau_{2}\right)}, 1\right)\right|
$$




$$
\begin{aligned}
& \leq \frac{\left(\tau_{2}-\tau_{1}\right) A\left(\tau_{1}, \tau_{2}\right)}{8 G^{2}\left(\tau_{1}, \tau_{2}\right)}\left[\frac{2|s|}{w+2}\left(G^{2(s-1)}\left(\tau_{2}, \frac{1}{\tau_{1}}\right)+\frac{w}{2} G^{s-\frac{1}{2}}\left(\tau_{2}, \frac{1}{\tau_{1}}\right)\right)\right. \\
& +\mathcal{F}_{\varkappa, \lambda}^{\aleph}\left(\frac{|s|}{w+2}\left(G^{2(s-1)}\left(\tau_{1}, \frac{1}{\tau_{2}}\right)+\frac{w}{2} G^{s-\frac{1}{2}}\left(\tau_{1}, \frac{1}{\tau_{2}}\right)\right)\right. \\
& \left.\left.-\frac{|s|}{w+2}\left(G^{2(s-1)}\left(\tau_{2}, \frac{1}{\tau_{1}}\right)+\frac{w}{2} G^{s-\frac{1}{2}}\left(\tau_{2}, \frac{1}{\tau_{1}}\right)\right)\right)\right] .
\end{aligned}
$$

Proof. Let $\zeta(\mu)=\frac{\mu^{s}+w \mu^{\frac{s}{2}}+1}{w+2}$ for $\mu>0$ and $s \notin(1,4)$.

$$
\zeta^{\prime}(\mu)=\frac{s}{w+2}\left(\mu^{s-1}+\frac{w}{2} \mu^{\frac{s}{2}-1}\right)
$$

By Corollary (3) . if follows that

$$
\begin{aligned}
& \left|\frac{1}{2}\left[\frac{\zeta\left(\frac{\tau_{2}}{\tau_{1}}\right)+\zeta\left(\frac{\tau_{1}}{\tau_{2}}\right)}{2}+\zeta\left(\frac{\frac{\tau_{2}}{\tau_{1}}+\frac{\tau_{1}}{\tau_{2}}}{2}\right)\right]-\frac{1}{\frac{\tau_{2}}{\tau_{1}}-\frac{\tau_{1}}{\tau_{2}}} \int_{\frac{\tau_{1}}{\tau_{2}}}^{\frac{\tau_{2}}{\tau_{1}}} \zeta(x) d x\right| \\
& =\mid \frac{1}{2}\left[\frac{1}{2}\left[\frac{\tau_{2}^{s}+w\left(\tau_{1} \tau_{2}\right)^{\frac{s}{2}}+\tau_{1}^{s}}{\tau_{1}^{s}(w+2)}+\frac{\tau_{1}^{s}+w\left(\tau_{1} \tau_{2}\right)^{\frac{s}{2}}+\tau_{2}^{s}}{\tau_{2}^{s}(w+2)}\right]\right. \\
& \left.+\frac{\left(\frac{\tau_{2}}{\tau_{1}}+\frac{\tau_{1}}{\tau_{2}}\right)^{s}+w\left(\frac{\tau_{2}}{\tau_{1}}+\frac{\tau_{1}}{\tau_{2}}\right)^{\frac{s}{2}}}{w+2}\right]-\frac{1}{w+2}\left[\frac{\left(\frac{\tau_{2}}{\tau_{1}}\right)^{s+1}-\left(\frac{\tau_{1}}{\tau_{2}}\right)^{s+1}}{(s+1)\left(\frac{\tau_{2}}{\tau_{1}}-\frac{\tau_{1}}{\tau_{2}}\right)}\right. \\
& \left.+w \frac{\left(\frac{\tau_{2}}{\tau_{1}}\right)^{\frac{s}{2}+1}-\left(\frac{\tau_{1}}{\tau_{2}}\right)^{\frac{s}{2}+1}}{\left(\frac{s}{2}+1\right)\left(\frac{\tau_{2}}{\tau_{1}}-\frac{\tau_{1}}{\tau_{2}}\right)}+1\right] \mid \\
& =\left|\frac{H_{w, s}^{s}\left(\tau_{1}, \tau_{2}\right)}{H\left(\tau_{1}^{s}, \tau_{2}^{s}\right)}+H_{w,\left(\frac{s}{2}+1\right)}^{\frac{s}{2}+1}\left(\frac{\tau_{2}}{\tau_{1}}+\frac{\tau_{1}}{\tau_{2}}, 1\right)-H_{w, s}^{s}\left(\frac{L\left(\tau_{1}^{2}, \tau_{2}^{2}\right)}{G^{2}\left(\tau_{1}, \tau_{2}\right)}, 1\right)\right| \\
& \times \frac{\frac{\tau_{2}}{\tau_{1}}-\frac{\tau_{1}}{\tau_{2}}}{16}\left[2\left|\zeta^{\prime}\right| \frac{\tau_{2}}{\tau_{1}}\left|+\mathcal{F}_{\varkappa, \lambda}^{\aleph}\left(\left|\zeta^{\prime}\left(\frac{\tau_{1}}{\tau_{2}}\right)\right|-\left|\zeta^{\prime}\left(\frac{\tau_{2}}{\tau_{1}}\right)\right|\right)\right|\right] \\
& =\frac{\tau_{2}^{2}-\tau_{1}^{2}}{16 \tau_{1} \tau_{2}}\left[2\left|\frac{s}{w+2}\left(\left(\frac{\tau_{2}}{\tau_{1}}\right)^{s-1}+\frac{w}{2}\left(\frac{\tau_{2}}{\tau_{1}}\right)^{\frac{s}{2}-1}\right)\right|\right. \\
& \left.+\mathcal{F}_{\varkappa, \lambda}^{\aleph}\left(\left|\frac{s}{w+2}\left(\left(\frac{\tau_{1}}{\tau_{2}}\right)^{s-1}+\frac{w}{2}\left(\frac{\tau_{1}}{\tau_{2}}\right)^{\frac{s}{2}-1}\right)\right|-\left|\frac{s}{w+2}\left(\left(\frac{\tau_{2}}{\tau_{1}}\right)^{s-1}+\frac{w}{2}\left(\frac{\tau_{2}}{\tau_{1}}\right)^{\frac{s}{2}-1}\right)\right|\right)\right] \\
& =\frac{\left(\tau_{2}-\tau_{1}\right) A\left(\tau_{1}, \tau_{2}\right)}{8 G^{2}\left(\tau_{1}, \tau_{2}\right)}\left[\frac{2|s|}{w+2}\left(G^{2(s-1)}\left(\tau_{2}, \frac{1}{\tau_{1}}\right)+\frac{w}{2} G^{s-\frac{1}{2}}\left(\tau_{2}, \frac{1}{\tau_{1}}\right)\right)\right. \\
& +\mathcal{F}_{\varkappa, \lambda}^{\aleph}\left(\frac{|s|}{w+2}\left(G^{2(s-1)}\left(\tau_{1}, \frac{1}{\tau_{2}}\right)+\frac{w}{2} G^{s-\frac{1}{2}}\left(\tau_{1}, \frac{1}{\tau_{2}}\right)\right)\right.
\end{aligned}
$$




$$
\left.\left.-\frac{|s|}{w+2}\left(G^{2(s-1)}\left(\tau_{2}, \frac{1}{\tau_{1}}\right)+\frac{w}{2} G^{s-\frac{1}{2}}\left(\tau_{2}, \frac{1}{\tau_{1}}\right)\right)\right)\right],
$$

obviously (47) and 48) yield 45).

\section{Conclusion}

In this paper, we have defined and proved some Hermite-Hadamard and Fejertype inequalities for generalize convex functions of Riana type. In addition, we find some interesting integral inequalities. All these results are new and amazing in literature. These results of the convex analysis are the basis and argument for many inequalities in pure and applied sciences. One thing to keep in mind, in the field of convex analysis and inequalities if we face problems, generalized notions and conceptions about convex functions are required to obtain pertinent and applicable results. It is high time to find the applications of these inequalities along with efficient numerical methods. We believe that our new results regarding generalize convex function of Raina type will have a very deep research in this fascinating field of inequalities and also in pure and applied sciences. The amazing techniques and wonderful ideas of this paper can be extended on the co-ordinates along with fractional calculus. In the future our goal is that we will continue our research work in this direction furthermore.

Author Contribution Statements All authors contributed equally to this work.

Declaration of Competing Interests The authors declare that they have no known competing financial interests or personal relationships that could have appeared to influence the work reported in this paper.

\section{REFERENCES}

[1] Butt, S. I., Nadeem, M., Qaisar, S., Akdemir, A. O., Abdeljawad, T., Hermite-Jensen-Mercer type inequalities for conformable integrals and related results, Adv. Differ. Equ., 1 (2020), 1-24. https://doi.org/10.1186/s13662-020-02968-4

[2] Chu, H. H., Kalsoom, H., Rashid, S., Idrees, M., Safdar, F., Chu, Y-M., Baleanu, D., Quantum analogs of Ostrowski type inequalities for Rainas function correlated with coordinated generalized $\eta$-convex functions, Symmetry, 12(2) (2020), 1-26. https://doi.org/10.3390/sym12020308

[3] Dragomir, S. S., Fitzpatrik, S., The Hadamard's inequality for $s$-convex functions in the second sense, Demonstratio Math., 32(4) (1999), 687-696. https://doi.org/10.1515/dema-19990403

[4] Eftekhari, N., Some remarks on $(s, m)$-convexity in the second sense, J. Math. Inequal., 8 (2014), 489-495. dx.doi.org/10.7153/jmi-08-36

[5] Fejér, L., Über die Fourierreihen, II. Math. Naturwiss. Anz Ungar. Akad. Wiss., 24 (1906).

[6] Hadamard, J., Étude sur les propriétés des fonctions entiéres en particulier d'une fonction considéréé par Riemann, J. Math. Pures. Appl., 58 (1893), 171-215. http://eudml.org/doc/234668 
[7] Hernández, H., Jorge, E., Vivas-Cortez, M., Hermite-Hadamard inequalities type for Raina's Fractional integral operator using $\eta$-convex functions, Revista de Mathemática Teoríay Aplicaciones., 26(1) (2019), 1-20. http://dx.doi.org/10.15517/rmta.v26i1.35515

[8] Khan, S., Khan, M. A., Butt, S. I., Chu, Y-M., A new bound for the Jensen gap pertaining twice differentiable functions with applications, Adv. Differ. Equ., 1 (2020), 1-11. https://doi.org/10.1186/s13662-020-02794-8

[9] Mehmood, N., Butt, S. I., Pečarić, D., Pečarić, J., Generalizations of cyclic refinements of Jensenâ's inequality by Lidstoneâ's polynomial with applications in Information Theory, $J$. Math. Inequal., 14(1) (2020), 249-271. dx.doi.org/10.7153/jmi-2020-14-17

[10] Niculescu, C. P., Persson, L. E., Convex Functions and Their Applications, Springer, New York, 2006. https://doi.org/10.1007/0-387-31077-0

[11] Özdemir, M. E., Yildiz, C., Akdemir, A. O., Set, E., On some inequalities for $s$-convex functions and applications, J. Ineq. Appl., 333 (2013), 2-11. https://doi.org/10.1186/1029242X-2013-333

[12] Raina, R. K., On generalized Wright's hypergeometric functions and fractional calculus operators, East Asian Math. J., 21(2) (2005), 191-203.

[13] Sarikaya, M. Z., Saglam, A., Yildirim, H., On some Hadamard type inequalities for $h$-convex functions, J. Math. Anal., 2(3) (2008), 335-341. https://doi.org/10.1186/s13660-019-2151-2

[14] Set, E., Noor, M. A., Awan, M. U., Gözpinar, A., Generalized Hermite-Hadamard type inequalities involving fractional integral operator, J. Inequal. Appl., 169 (2017), 1-10. https://doi.org/10.1186/s13660-017-1444-6

[15] Set, E., Some new generalizations of Ostrowski type inequalities for $s$-convex functions via fractional integral operators, Filomat., 32(16) (2018), 5595-5609. https://doi.org/10.2298/FIL1816595S

[16] Toader, G., Some generalizations of the convexity, Proceedings of The Colloquium on Approximation and Optimization, Univ. Cluj-Napoca, Cluj-Napoca, (1985), 329-338.

[17] Xi, B. Y., Q, F., Some integral inequalities of Hermite-Hadamard type for convex functions with applications to means, J. Funct. Spaces. Appl., 2012 Article ID 980438, (2012), 1-14. https://doi.org/10.1155/2012/980438

[18] Butt, S. I., Tariq, M., Aslam, A., Ahmad, H., Nofal, T. A., Hermite-Hadamard type inequalities via generalized harmonic exponential convexity and applications, Journal of Function Spaces, 2021 Article ID 5533491 (2021), 12 pages. https://doi.org/10.1155/2021/5533491

[19] Butt, S. I., Kashuri, A., Tariq, M., Nasir, J., Aslam, A., Gao, W., n-polynomial exponential type p-convex function with some related inequalities and their applications, Heliyon, 6(11) (2020), e05420 ISSN 2405-8440. https://doi.org/10.1016/j.heliyon.2020.e05420

[20] Butt, S. I., Kashuri, A., Tariq, M., Nasir, J., Aslam, A., Gao, W., Hermite-Hadamard-type inequalities via n-polynomial exponential-type convexity and their applications, Adv. Differ. Equ., 508 (2020). https://doi.org/10.1186/s13662-020-02967-5

[21] Gao, W., Kashuri, A., Butt, S. I., Tariq, M., Aslam, A., Nadeem, M., New inequalities via npolynomial harmonically exponential type convex functions, AIMS Mathematics, 5(6) (2020), 6856-6873. doi: 10.3934/math.2020440

[22] Butt, S. I., Kashuri, A., Umar, M., Aslam, A., Gao, W., Hermite-Jensen-Mercer type inequalities via $\psi$-Riemann-Liouville k-fractional integrals, AIMS Mathematics, 5(5) (2020), 5193-5220. doi:10.3934/math.2020334 